\title{
Analysis aspects of Willmore surfaces
}

\section{Journal Article}

\section{Author(s):}

Rivière, Tristan

Publication date:

2008-10-01

Permanent link:

https://doi.org/10.3929/ethz-b-000072456

Rights / license:

In Copyright - Non-Commercial Use Permitted

\section{Originally published in:}

Inventiones mathematicae 174(1), https://doi.org/10.1007/s00222-008-0129-7 


\section{Analysis aspects of Willmore surfaces}

\section{Tristan Rivière}

Department of Mathematics, ETH Zentrum, CH-8093 Zürich, Switzerland

(e-mail: riviere@math.ethz.ch)

Oblatum 31-III-2007 \& 4-III-2008

Published online: 10 April 2008 - (C) Springer-Verlag 2008

Abstract. A new formulation for the Euler-Lagrange equation of the Willmore functional for immersed surfaces in $\mathbb{R}^{m}$ is given as a nonlinear elliptic equation in divergence form, with non-linearities comprising only Jacobians. Letting $\vec{H}$ be the mean curvature vector of the surface, our new formulation reads $\mathcal{L} \vec{H}=0$, where $\mathcal{L}$ is a well-defined locally invertible self-adjoint elliptic operator. Several consequences are studied. In particular, the long standing open problem asking for a meaning to the Willmore Euler-Lagrange equation for immersions having only $L^{2}$-bounded second fundamental form is now solved. The regularity of weak Willmore immersions with $L^{2}$-bounded second fundamental form is also established. Its proof relies on the discovery of conservation laws which are preserved under weak convergence. A weak compactness result for Willmore surfaces with energy less than $8 \pi$ (the Li-Yau condition ensuring the surface is embedded) is proved, via a point removability result established for Wilmore surfaces in $\mathbb{R}^{m}$, thereby extending to arbitrary codimension the main result in [KS3]. Finally, from this point-removability result, the strong compactness of Willmore tori below the energy level $8 \pi$ is proved both in dimension 3 (this had already been settled in [KS3]) and in dimension 4 .

\section{Introduction}

Not only do weak formulations of partial differential equations offer the possibility to enlarge the class of solutions to the space of singular solutions, but they further provide a flexible setting in which the analysis of smooth solutions becomes far more efficient. This is the idea which we will illustrate in this paper by introducing a new weak formulation for Willmore surfaces.

Let $\Sigma$ be a given oriented surface, and $\vec{\Phi}$ be a smooth positive immersion of $\Sigma$ into the Euclidean space $\mathbb{R}^{m}$, for some $m \geq 3$. We introduce the Gauss map $\vec{n}$ from $\Sigma$ into $G r_{m-2}\left(\mathbb{R}^{m}\right)$, the Grassmanian of oriented $(m-2)$-planes 
of $\mathbb{R}^{m}$, which to every point $x$ in $\Sigma$ assigns the unit $(m-2)$-vector defining the $(m-2)$-plane $N_{\vec{\Phi}(x)} \vec{\Phi}(\Sigma)$ orthogonal to the oriented tangent space $T_{\vec{\Phi}(x)} \vec{\Phi}(\Sigma)$. The map $\vec{n}$ induces a projection map $\pi_{\vec{n}}$; namely, for every vector $\xi$ in $T_{\vec{\Phi}(x)}\left(\mathbb{R}^{m}\right), \pi_{\vec{n}}(\xi)$ is the orthogonal projection of $\xi$ onto $N_{\vec{\Phi}(x)} \vec{\Phi}(\Sigma)$. Let now $\vec{B}_{x}$ be the second fundamental form of the immersion $\vec{\Phi}$. It is a symmetric bilinear form on $T_{x} \Sigma$ with values in $N_{\vec{\Phi}(x)} \vec{\Phi}(\Sigma)$, explicitly given by $\vec{B}_{x}=\pi_{\vec{n}} \circ d^{2} \vec{\Phi}$. Using the ambient scalar product in $\mathbb{R}^{m}$, we define the trace of $\vec{B}_{x}$, namely Here $\left\{e_{1}, e_{2}\right\}$ is an arbitrary orthonormal basis of $T_{x} \Sigma$. The mean curvature vector $\vec{H}(x)$ at $x$ is the vector in $N_{\vec{\Phi}(x)} \vec{\Phi}(\Sigma)$ given by

$$
\vec{H}(x)=\frac{1}{2} \operatorname{tr}\left(\vec{B}_{x}\right) .
$$

Note that when $m=3$, one finds $\vec{H}=H \vec{n}$, where $\vec{n}$ is the unit normal vector, and $H=\left(\kappa_{1}+\kappa_{2}\right) / 2$ is the average of the principal curvatures $\kappa_{1}, \kappa_{2}$ of the surface at $\vec{\Phi}(x)$.

Equipped with the aforementioned notation, the Willmore functional is the Lagrangian

$$
W(\vec{\Phi}(\Sigma)):=\int_{\Sigma}|\vec{H}|^{2} d v l_{g},
$$

where $d$ vol $_{g}$ is the area form of the metric $g$ induced on $\vec{\Phi}(\Sigma)$ by the canonical metric on $\mathbb{R}^{m}$.

This Lagrangian was seemingly first considered in the early twentieth century in various works by Thomsen [Tho] and Schadow, and subsequently by Blaschke [Bla]. It was reintroduced, and more systematically studied within the framework of the conformal geometry of surfaces, by Willmore in 1965 [Wil]. The Willmore functional also plays an important role in various areas of science. In molecular biology, it is known as the Helfrich Model [Hef], where it appears as a surface energy for lipid bilayers. In solid mechanics, the Willmore functional arises as the limit-energy for thinplate theory (see [FJM]). Finally, in general relativity, the Lagrangian (I.1) appears as the main term in the expression of the Hawking quasilocal mass (see $[\mathrm{Haw}]$ and $[\mathrm{HI}]$ ).

The generic nature of the Willmore functional is partly due to its invariance under conformal transformations of the metric of the ambient space. This amazing property was first brought into light by White [Whi] in the case when $m=3$; and it was subsequently generalized by B.Y. Chen [Che].

In the present paper, we are interested in studying the critical points of (I.1) for perturbations of the form $\vec{\Phi}+t \vec{\xi}$, where $\vec{\xi}$ is an arbitrary smooth map on $\Sigma$ into $\mathbb{R}^{m}$. These critical points are known as Willmore surfaces. The aforementioned property of the Willmore functional guarantees that Willmore surfaces remain Willmore through conformal transformations of $\mathbb{R}^{m}$. Clearly, any minimal surface, i.e. one for which $\vec{H} \equiv 0$, is a Willmore surface. In fact, any minimal surface realizes an absolute minimum of $W$. Many 
more Willmore surfaces are known, and works specialized with producing such surfaces are profuse (see for examples [Wil2] and [PS]).

Willmore, in the case $m=3$, and thereafter Weiner [Wei], for general $m$, established that Willmore surfaces satisfy an Euler-Lagrange equation ${ }^{1}$. Prior to stating this equation, we need to introduce some notation. Given any vector $\vec{w}$ in $N_{\vec{\Phi}(x)} \vec{\Phi}(\Sigma)$, consider the symmetric endomorphism $A_{x}^{\vec{w}}$ of $T_{x} \Sigma$ satisfying $g\left(A_{x}^{\vec{w}}(\vec{X}), \vec{Y}\right)=B_{x}(\vec{X}, \vec{Y}) \cdot \vec{w}$, where $\cdot$ denotes the standard scalar product in $\mathbb{R}^{m}$, for every pair of vectors $\vec{X}$ and $\vec{Y}$ in $T_{x} \Sigma$. The map $A_{x}: \vec{w} \mapsto A_{x}^{\vec{w}}$ is a homomorphism from $N_{\vec{\Phi}(x)} \vec{\Phi}(\Sigma)$ into $S_{g} \Sigma_{x}$, the linear space of symmetric endomorphisms on $T_{x} \Sigma$. We next define $\tilde{A}_{x}={ }^{t} A_{x} \circ A_{x}$, which is an endomorphism of $N_{\vec{\Phi}(x)} \vec{\Phi}(\Sigma)$. If $\left\{\vec{e}_{1}, \vec{e}_{2}\right\}$ is an orthonormal basis of $T_{x} \Sigma$, and if $\vec{L}$ is a vector in $N_{\vec{\Phi}(x)} \vec{\Phi}(\Sigma)$, then it is readily seen that $\tilde{A}(\vec{L})=\sum_{i, j} \vec{B}_{x}\left(\vec{e}_{i}, \vec{e}_{j}\right) \vec{B}_{x}\left(\vec{e}_{i}, \vec{e}_{j}\right) \cdot \vec{L}$. With this notation, $\vec{\Phi}$ is a smooth Willmore immersion if and only if it satisfies Euler-Lagrange equation

$$
\Delta_{\perp} \vec{H}-2|\vec{H}|^{2} \vec{H}+\tilde{A}(\vec{H})=0,
$$

where $\Delta_{\perp}$ is the negative covariant Laplacian for the connection $D$ in the normal bundle $N \vec{\Phi}(\Sigma)$ derived from the ambient scalar product in $\mathbb{R}^{m}$. Namely, for every section $\sigma$ of $N \vec{\Phi}(\Sigma)$, one has $D_{\vec{X}} \sigma:=\pi_{\vec{n}}\left(\sigma_{*} \vec{X}\right)$. Note that when $m=3$, since $\vec{H}=H \vec{n}$, (I.2) becomes

$$
\Delta_{g} H+2 H\left(|H|^{2}-K\right)=0,
$$

where $\Delta_{g}$ is the negative Laplace operator for the induced metric $g$ on $\vec{\Phi}(\Sigma)$, while $K$ is the scalar curvature of $(\vec{\Phi}(\Sigma), g)$.

Despite their elegant aspect, (I.2) and (I.3) yield challenging mathematical difficulties. Observe indeed that the highest order term $\Delta_{\perp} \vec{H}$ in (I.2) (resp. $\Delta_{g} H$ in (I.3)) is nonlinear, since the metric $g$ defining the Laplace operator depends on the immersion $\vec{\Phi}$. One further difficulty is embodied by the "incompatibilty" between the Euler-Lagrange equations (I.2) and (I.3) and the Lagrangian (I.1). More precisely, the minimal regularity assumption ensuring that the Lagrangian (I.1) is finite, namely that the second fundamental form $\vec{B}$ be square-integrable on $\vec{\Phi}(\Sigma)$, is insufficient for the nonlinearities in (I.2) and (I.3) to have a distributional meaning: the expression $|\vec{H}|^{2} \vec{H}$ requires at least that $\vec{H}$ be in $L^{3}$.

Recently, the author proved in [Ri1] that any Euler-Lagrange equation arising from a two-dimensional conformally invariant Lagrangian with quadratic growth (e.g. the harmonic map equation into a Riemannian submanifold and the prescribed mean curvature equation) can be written in divergence form. This feature has numerous consequences for the analysis of

1 This equation was allegedly already known to Thomsen, Schadow, and Blaschke; thus several decades before Willmore and Weiner "revived" it. 
the Euler-Lagrange equation. It allows in particular to extend the set of solutions to subspaces of distributions with very low regularity requirements. The results developed in [Ri1] hinted that they could be extended to other conformally invariant equations such as the harmonic map equations into Lorentzian manifolds. Considering in addition the correspondence established by Bryant [Bry] between Willmore surfaces in $\mathbb{R}^{3}$ and harmonic maps into the Minkowski sphere $S^{3,1} \subset \mathbb{R}^{4,1}$, the author found a strong encouragement for seeking a divergence form for the Willmore Euler-Lagrange equation (I.1).

We now state the first main result of this paper.

Theorem I.1 The Willmore Euler-Lagrange equation (I.2) is equivalent to

$$
d\left(*_{g} d \vec{H}-3 *_{g} \pi_{\vec{n}}(d \vec{H})\right)-d \star(d \vec{n} \wedge \vec{H})=0,
$$

where $*_{g}$ is the Hodge operator on $\Sigma$ associated to the induced metric g, and where $\star$ is the Hodge operator on p-vectors in $\mathbb{R}^{m}$ defined as follows. If $(\alpha, \beta)$ is a pair of $p$-vectors in $\mathbb{R}^{m}$, one has

$$
\alpha \wedge \star \beta=\langle\alpha, \beta\rangle \star 1,
$$

where $\langle\alpha, \beta\rangle$ is the scalar product for the canonical metric in $\mathbb{R}^{m}$. In particular, $\star 1$ is the positively oriented unit $m$-vector in $\mathbb{R}^{m}$.

In particular, a conformal immersion $\vec{\Phi}$ from the flat disc $D^{2}=\Sigma$ into $\mathbb{R}^{m}$ is Willmore if and only if

$$
\Delta \vec{H}-3 \operatorname{div}\left(\pi_{\vec{n}}(\nabla \vec{H})\right)+\operatorname{div} \star\left(\nabla^{\perp} \vec{n} \wedge \vec{H}\right)=0,
$$

where the operators $\Delta$, div, $\nabla$, and $\nabla^{\perp}$ are understood with respect to the flat metric on $D^{2}$. Namely, $\Delta=\partial_{x_{1}}^{2}+\partial_{x_{2}}^{2}$, div $=\operatorname{tr} \circ \nabla, \nabla=\left(\partial_{x_{1}}, \partial_{x_{2}}\right)$, and $\nabla^{\perp}=\left(-\partial_{x_{2}}, \partial_{x_{1}}\right)$.

Note that owing to the conformal invariance of our problem, it is sufficient to prove (I.5). The more general version (I.4) may then be deduced through a change of coordinates.

This first result justifies the following terminology: for a given map $\vec{n}$ from $D^{2}$ into $G_{m-2}\left(\mathbb{R}^{m}\right)$, we shall denote the Willmore operator by $\mathcal{L}_{\vec{n}}$, which is the operator that to a function $\vec{w}$ from $D^{2}$ into $\mathbb{R}^{m}$ assigns

$$
\mathcal{L}_{\vec{n}} \vec{w}:=\Delta \vec{w}-3 \operatorname{div}\left(\pi_{\vec{n}}(\nabla \vec{w})\right)+\operatorname{div} \star\left(\nabla^{\perp} \vec{n} \wedge \vec{w}\right) .
$$

Although this is not difficult to verify, it is somewhat a good surprise that this elliptic operator is self-adjoint: for any choice of map $\vec{n}$ in $W^{1,2}\left(D^{2}, G r_{m-2}\left(\mathbb{R}^{m}\right)\right)$ and for any choice of compactly supported maps $\vec{v}$ and $\vec{w}$ from $D^{2}$ into $\mathbb{R}^{m}$, there holds:

$$
\int_{D^{2}} \vec{v} \cdot \mathscr{L}_{\vec{n}} \vec{w}=\int_{D^{2}} \mathcal{L}_{\vec{n}} \vec{v} \cdot \vec{w} .
$$


Another important quantity is

$$
\pi_{\vec{n}}(\vec{v}):=\vec{n}\llcorner(\vec{n}\llcorner\vec{v}),
$$

where $\mathrm{L}$ is the interior multiplication between $q$-vectors and $p$-vectors, with $q \geq p$, producing $(q-p)$-vectors in $\mathbb{R}^{m}$ (for details, see [Fe, 1.5.1 and 1.7.5]). For every choice of $q, p$, and ( $m-q+p)$-forms, respectively $\alpha, \beta$, and $\gamma$, we have:

$$
\langle\alpha\llcorner\beta, \gamma\rangle=\langle\alpha, \beta \wedge \gamma\rangle .
$$

With this notation, we infer

$$
\operatorname{div}\left(\pi_{\vec{n}}(\nabla \vec{w})\right)=\Delta[\vec{n}\llcorner(\vec{n}\llcorner\vec{w}(x))]-\vec{n}\llcorner(\nabla \vec{n}\llcorner\vec{w}(x))-\nabla \vec{n}\llcorner(\vec{n}\llcorner\vec{w})(x) .
$$

When the unit ( $m-2$ )-vector $\vec{n}$ belongs to $W^{1,2}$, the distribution $\mathcal{L}_{\vec{n}} \vec{w}$ given by (I.6) is well-defined for any $\vec{w}$ in $L^{2}\left(D^{2}\right)$. Thus our new formulation reconciles the Euler-Lagrange equations (I.4) and (I.5) with the Lagrangian (I.1). Indeed, the former have a distributional meaning under the least possible regularity requirement for the immersion $\vec{\Phi}(\Sigma)$; namely that the Gauss map be in $W^{1,2}$ with respect to the induced metric.

Before giving a weak formulation to Willmore immersions, we first introduce the notion of Weak immersion with $L^{2}$-bounded second fundamental form.

\section{Definition I.1 (Weak immersions with $L^{2}$-bounded second fundamen-} tal form) Let $\vec{\Phi}$ be a $W^{1,2}$-map from a two-dimensional manifold $\Sigma$ into $\mathbb{R}^{m}$. $\vec{\Phi}$ is called a weak immersion with locally $L^{2}$-bounded second fundamental form if for every $x \in \Sigma$ there exists an open disk $D$ in $\Sigma$, a constant $C>0$, and a sequence of smooth embeddings $\vec{\Phi}^{k}$ from $D^{2}$ into $\mathbb{R}^{m}$, such that

$$
\mathscr{H}^{2}(\vec{\Phi}(D)) \neq 0
$$

ii)

iii)

$$
\begin{gathered}
\mathscr{H}^{2}\left(\vec{\Phi}^{k}(D)\right) \leq C<+\infty \\
\int_{D}\left|B^{k}\right|^{2} d \text { vol }_{g^{k}} \leq \frac{8 \pi}{3}
\end{gathered}
$$

$$
\vec{\Phi}^{k} \rightarrow \vec{\Phi} \quad \text { weakly in } W^{1,2},
$$

where $\mathscr{H}^{2}$ is the two-dimensional Hausdorff measure, $B^{k}$ is the second fundamental form associated to the embedding $\vec{\Phi}^{k}$, and $g^{k}$ denotes the metric on $\vec{\Phi}^{k}(\Sigma)$ obtained via the pull-back by $\vec{\Phi}^{k}$ of the induced metric.

For example, $W^{2,2}$ graphs in $\mathbb{R}^{3}$ of maps from $\mathbb{R}^{2}$ into $\mathbb{R}$ are weak immersions with $L^{2}$-bounded second fundamental form. 
The following result was established by F. Hélein (cf. Theorem 5.1.1 in [Hel]), thereby generalizing a result of T. Toro [To1], [To2], and of S. Müller and V. Sveràk [MS].

Theorem I.2 (Existence of local conformal coordinates for weak immersions) Let $\vec{\Phi}$ be a weak immersion from a two-dimensional manifold $\Sigma$ into $\mathbb{R}^{m}$ with $L^{2}$-bounded second fundamental form. Then for every $x$ in $\Sigma$, there exist an open disk $D$ in $\Sigma$ containing $x$ and a homeomorphism $\Psi$ of $D$ such that $\vec{\Phi} \circ \Psi$ is a conformal bilipschitz immersion. The metric $g$ on $D$ induced by the standard metric of $\mathbb{R}^{m}$ is continuous in this parametrization. Moreover, the Gauss map $\vec{n}$ of this immersion is in $W^{1,2}\left(D, G r_{m-2}\left(\mathbb{R}^{m}\right)\right)$ relative to the induced metric $g$.

We are now ready to define the notion of weak Willmore immersion with $L^{2}$-bounded second fundamental form.

Definition I.2 (Weak Willmore immersions with $L^{2}$-bounded second fundamental form) A weak immersion $\vec{\Phi}$ from a two-dimensional manifold $\Sigma$ into $\mathbb{R}^{m}$ with $L^{2}$-bounded second fundamental form is Willmore when

$$
\Delta \vec{H}-3 \operatorname{div}\left(\pi_{\vec{n}}(\nabla \vec{H})\right)+\operatorname{div} \star\left(\nabla^{\perp} \vec{n} \wedge \vec{H}\right)=0 \quad \text { in } \mathscr{D}^{\prime}\left(D^{2}\right)
$$

holds about every point $x \in \Sigma$ in a conformal parametrization from the two-dimensional disk $D^{2}$, as indicated in Theorem I.2. The operators $\Delta$, div, $\nabla$, and $\nabla^{\perp}$ are as in Theorem I.1.

Observe that this definition is sensible, since, as previously noted, the expression

$$
\nabla \vec{H}-3 \pi_{\vec{n}}(\nabla \vec{H})+\star\left(\nabla^{\perp} \vec{n} \wedge \vec{H}\right)
$$

has a distributional meaning so soon as the Gauss map $\vec{n}$ lies in $W^{1,2}$. Note also that the notion of $W^{2,2}$ Willmore graph in $\mathbb{R}^{3}$ naturally ensues from Definition I.2.

We next state the second main result of the present paper.

Theorem I.3 (Regularity for weak Willmore immersions) Let $\vec{\Phi}$ be a weak Willmore immersion from a two-dimensional manifold $\Sigma$ into $\mathbb{R}^{m}$ with $L^{2}$-bounded second fundamental form. Then $\vec{\Phi}(\Sigma)$ is the image of a real analytic immersion.

As we shall discover in the sequel, the following conservation laws play a central role in proving the above regularity result.

Theorem I.4 (Conservation laws for weak Willmore immersions) Let $\vec{\Phi}$ be a weak Willmore immersion from the flat diskhv- $D^{2}$ into $\mathbb{R}^{m}$ with $L^{2}$-bounded second fundamental form. Suppose that $\vec{\Phi}$ is conformal. We 
let $\vec{L}$ be the map from $D^{2}$ into $\mathbb{R}^{m}$ satisfying

$$
\nabla^{\perp} \vec{L}:=\nabla \vec{H}-3 \pi_{\vec{n}}(\nabla \vec{H})+\star\left(\nabla^{\perp} \vec{n} \wedge \vec{H}\right) .
$$

Then there holds

$$
\nabla \vec{\Phi} \cdot \nabla^{\perp} \vec{L}=0
$$

and

$$
\nabla \vec{\Phi} \wedge \nabla^{\perp} \vec{L}=2(-1)^{m} \nabla\left[\star ( \vec { n } \llcorner \vec { H } ) ] \left\llcorner\nabla^{\perp} \vec{\Phi} .\right.\right.
$$

Furthermore, denoting respectively by $S$ and $\vec{R}$ the scalar and the 2-vector-valued function on $D^{2}$ implicitly defined via $\nabla S:=\vec{L} \cdot \nabla \vec{\Phi}$ and $\nabla \vec{R}:=$ $\nabla \vec{\Phi} \wedge \vec{L}+2(-1)^{m}[\star(\vec{n}\llcorner\vec{H})]\llcorner\nabla \vec{\Phi}$, one finds that

$$
\nabla^{\perp} \vec{R}=(-1)^{m-1} \star(\vec{n} \bullet \nabla \vec{R})+\star \vec{n} \nabla S .
$$

Here $\bullet$ is the first order contraction between multivectors. It satisfies $\alpha \bullet \beta=\alpha \mathrm{L} \beta$ when $\beta$ is a 1-vector; and $\alpha \bullet(\beta \wedge \gamma)=(\alpha \bullet \beta) \wedge \gamma+$ $(-1)^{p q}(\alpha \bullet \gamma) \wedge \beta$ when $\beta$ and $\gamma$ are respectively any $p$-vector and any $q$-vector.

Let us note that (I.13) implies that on $D^{2}$ there holds

$$
\Delta \vec{R}=(-1)^{m-1} \star \nabla^{\perp} \vec{n} \bullet \nabla \vec{R}+\nabla^{\perp}(\star \vec{n}) \cdot \nabla S .
$$

Owing to the isotropy of the Euclidean space, the operators $\wedge, \star$, L, and $\bullet$ all commute with differentiation in $D^{2}$. Accordingly, the identities (I.11) and (I.12) express the vanishing of linear combinations of certain Jacobians. Thanks to this very special structure, it will be possible to pass to the limit under weak convergence of Willmore surfaces having uniformly bounded Willmore energies. This observation also justifies the use of the term "conservation laws" to describe (I.11) and (I.12). Interestingly enough, it is shown in [BR] that these conservation laws are in fact equivalent to some conformal Willmore Euler-Lagrange equation which correponds to critical points to $W$ for a fixed conformal structure. This remarkable feature enables in particular to infer that weak limits of Willmore equations are themselves Willmore.

Equation (I.14) shows that the Laplacian of $\vec{R}$ arises as a linear combination of Jacobians. With the help of Wente-type estimates (such as those described in [Hel]), this important fact will be used in Sect. III to deduce that the Gauss map $\vec{n}$ is continuous. In turn, the continuity of the Gauss map yields the following $\epsilon$-regularity theorem ${ }^{2}$, which itself implies the regularity of weak Willmore immersions stated in Theorem I.3.

Theorem I.5 ( $\epsilon$-regularity for weak Willmore immersions) Let $\vec{\Phi}$ be a weak Willmore immersion from the unit two-dimensional disk $D^{2}$ into $\mathbb{R}^{m}$ with $L^{2}$-bounded second fundamental form. There exists a constant $\varepsilon>0$,

2 This result is established through different means in [KS1] for smooth Willmore surfaces. 
independent of $\vec{\Phi}$, with the following property. Let $\vec{n}$ be the Gauss map corresponding to the weak immersion $\vec{\Phi}$. We assume that $\vec{\Phi}$ is the bilipschitz parametrization given by Theorem I.2. If

$$
\int_{D^{2}}|\nabla \vec{n}|^{2} d v^{2} l_{g} \leq \varepsilon
$$

then for every $k \in \mathbb{N}$, there is a positive constant $C_{k}$ depending only on $k$, and such that

$$
\left\|\nabla^{k} \vec{n}\right\|_{L^{\infty}\left(D_{1 / 2}^{2}\right)}^{2} \leq C_{k} \int_{D^{2}}|\nabla \vec{n}|^{2} d \operatorname{vol}_{g}
$$

Here $D_{1 / 2}^{2}$ is the disk of radius $1 / 2$ in the flat metric on $D^{2}$.

Another fundamental task consists in describing the "boundary" of the moduli space of closed Willmore surfaces with given genus and bounded Willmore energy. To this end, one aims at describing the limiting behavior of sequences of Willmore surfaces $S_{n}$ with fixed topology and bounded Willmore energy. Modulo the action of the Möbius group of conformal transformations of $\mathbb{R}^{m}$ (which preserve the Willmore Lagrangian, and therefore the Willmore equation (I.4)), it is always possible to fix the area of each $S_{n}$ to be equal to 1 . Then, using a Federer/Fleming-type argument, a subsequence is extracted such that the current of integration on $S_{n}$ converges for the Flat topology to some limiting integral current of integration $S$ (refer to [Fe] for the terminology on integral currents). Because $S_{n}$ has a uniformly bounded Willmore energy and a fixed topology, the $L^{2}$ norm (relative to the induced metric) of its second fundamental form, and hence the $W^{1,2}$ norm of the Gauss map over the surface, are bounded. Calling upon Theorem I.5, a classical argument of compactness concentration allows to deduce that $S_{n}$ converges to $S$, in a suitable parametrization and in the $C^{k}$-topology, outside of finitely many points $\left\{p_{1}, \ldots, p_{k}\right\}$. This strong convergence implies that $S$ is a smooth Willmore surface a-priori outside of these points. It then appears natural to ask whether these singular points are "removable". This is the content of the following theorem, which extends to arbitrary codimension the main result in [KS3].

Theorem I.6 (Point removability for Willmore immersions) Let $\vec{\Phi}$ be a continuous map from $D^{2}$ into $\mathbb{R}^{m}$ with $\vec{\Phi}(0)=x_{0}$. Assume that $\vec{\Phi}$ realizes a finite-area Willmore immersion over $D^{2} \backslash\{0\}$ and that the $W^{1,2}$ energy of the Gauss map on $D^{2} \backslash\{0\}$ is bounded. Let $\mu$ be the restriction to $\vec{\Phi}\left(D^{2}\right)$ of the two-dimensional Hausdorff measure $\mathscr{H}^{2}$ in $\mathbb{R}^{m}$ weighed by the multiplicity function from $\vec{\Phi}\left(D^{2}\right)$ into $\mathbb{N}$ which to each point in $\vec{\Phi}\left(D^{2}\right)$ assigns its number of preimages under $\vec{\Phi}$. Suppose that $x_{0}$ has density less than 2:

$$
\liminf _{r \rightarrow 0} \frac{\mu\left(B_{r}^{m}\left(x_{0}\right)\right)}{\pi r^{2}}<2 .
$$


Then $\vec{\Phi}\left(D^{2}\right)$ is a $C^{1, \alpha}$-submanifold of $\mathbb{R}^{m}$ for every $\alpha<1$. Moreover, if $\vec{H}$ denotes the mean curvature vector of this submanifold, then there exists a constant vector $\vec{H}_{0}$ such that $\vec{H}(x)-\vec{H}_{0} \log \left|x-x_{0}\right|$ is a $C^{0, \alpha}$-function on $\vec{\Phi}\left(D^{2}\right)$. Here $\left|x-x_{0}\right|$ denotes the distance in $\vec{\Phi}\left(D^{2}\right)$ between $x$ and $x_{0}$. When $\vec{H}_{0}=0, \vec{\Phi}$ is an analytic Willmore immersion on the whole $D^{2}$.

We remind the reader that Bryant [Bry] has produced counterexamples to the above removability result when (I.17) is weakened to

$$
\liminf _{r \rightarrow 0} \frac{\mu\left(B_{r}^{m}\left(x_{0}\right)\right)}{\pi r^{2}}=2 .
$$

Assuming the validity of Theorem I.6 when $m=3$, Kuwert and Schätzle were able to establish the fact that the limit $S$ of a sequence of smooth Willmore surfaces $S_{n}$ is again a smooth Willmore submanifold in $\mathbb{R}^{3}$, under the hypothesis that the Willmore energy of each $S_{n}$ is less than $8 \pi-\delta$, for any fixed $\delta>0$. This last assumption ensures that $S$ is a graph about each $p_{i}$, $i=1, \ldots, k$, and that the residue $\vec{H}_{0}$ vanishes at every $p_{i}$. A careful inspection of the argument leading to these consequences (cf. p. 344 in [KS3]) reveals that the restriction to codimension 1 is in fact unnecessary.

The removability Theorem I.6 yields the last main result of this paper.

Theorem I.7 (Weak compactness of Willmore surfaces below 8 $\pi$ ) Let $m>2$ be an arbitrary integer, and let $\delta>0$. Consider a sequence $S_{n} \subset \mathbb{R}^{m}$ of smooth closed Willmore embeddings with uniformly bounded topology, unit area, and Willmore energy $W\left(S_{n}\right)$ bounded above by $8 \pi-\delta$. Assume further that $S_{n}$ converges weakly as varifolds to some limit $S$ which realizes a non-zero current. Then $S$ is itself a smooth Willmore embedding.

A theorem of Montiel ${ }^{3}$ [Mon] states that any non-umbillic Willmore 2 -sphere in $\mathbb{R}^{4}$ has Willmore energy larger than $8 \pi$. This result along with an argument from [KS3, cf. pp. 350-351] may be combined to our removability theorem to produce

Theorem I.8 (Strong compactness of Willmore tori with energy below $8 \pi$ ) Let $m=3$ or $m=4$, and let $\delta>0$ be arbitrary. The space of Willmore tori embedded in $\mathbb{R}^{m}$ having Willmore energy less that $8 \pi-\delta$ is compact - up to Möbius transformations - under smooth convergence of compactly contained surfaces in $\mathbb{R}^{m}$.

This result extends to dimension 4 Theorem 5.3 from [KS3], which is established in dimension 3.

A central role in the analysis of Willmore surfaces developed in this paper is played by the following observation, which, for the sake of brevity, is presented only for $m=3$. Consider an $L^{2}$ map $\vec{w}$ from $D^{2}$ into $\mathbb{R}^{3}$. We assume that it lies in the kernel of the Willmore operator $\mathcal{L}_{\vec{n}}$, where $\vec{n}$ is

\footnotetext{
${ }^{3}$ Already known in $\mathbb{R}^{3}$ from the work of Bryant [Bry].
} 
some map in $W^{1,2}\left(D^{2}, S^{2}\right)$; namely $\mathcal{L}_{\vec{n}} \vec{w}=0$. We introduce the following Hodge decomposition:

$$
\nabla \vec{w}-3 \pi_{N}(\nabla \vec{w})=\nabla \vec{A}+\nabla^{\perp} \vec{B},
$$

with the boundary condition $\vec{A}=0$ on $\partial D^{2}$. Let $\left\{\vec{\epsilon}_{1}, \vec{\epsilon}_{2}, \vec{\epsilon}_{3}\right\}$ be the canonical basis of $\mathbb{R}^{3}$. Then $\vec{A}$ and $\vec{B}=\sum_{i=1}^{3} B_{i} \vec{\epsilon}_{i}$ satisfy:

$$
\left\{\begin{aligned}
\Delta \vec{A} & =\nabla \vec{H} \wedge \nabla^{\perp} \vec{n}=\partial_{x_{2}} \vec{H} \wedge \partial_{x_{1}} \vec{n}-\partial_{x_{1}} \vec{H} \wedge \partial_{x_{2}} \vec{n} \\
\Delta B_{i} & =3 \operatorname{div}\left(\pi_{N}\left(\nabla^{\perp} \vec{w}\right)\right)=3 \sum_{j=1}^{3} \nabla\left(n_{i} n_{j}\right) \cdot \nabla^{\perp} w_{j} \\
& =3 \sum_{j=1}^{3} \partial_{x_{2}}\left(n_{i} n_{j}\right) \partial_{x_{1}} w_{j}-\partial_{x_{1}}\left(n_{i} n_{j}\right) \partial_{x_{2}} w_{j},
\end{aligned}\right.
$$

where $\vec{w}=\sum_{i=1}^{3} w_{i} \vec{\epsilon}_{i}$ and $\vec{n}=\sum_{i=1}^{3} n_{i} \vec{\epsilon}_{i}$. The system (I.20) bears a striking feature: all of the involved nonlinearities are linear combinations of Jacobians! Such a special algebraic structure plays a distinctive role in geometric analysis. This was probably first discovered by Wente [Wen]. In [Hel], Hélein gives a detailed account of the consequence borne by those "Jacobian structures". In the present work, we shall make extensive use of Wente-type estimates.

Final remarks i) The analysis which we are developing in this paper points to a new direction for reaching a new proof of Simon's result [Si2] on the existence of embedded energy-minimizing Willmore tori in $\mathbb{R}^{m}$, for every $m \geq 3$ (see [BR]).

ii) Our approach should be particularly helpful in the study of the Willmore flow, as initiated in the works of Kuwert and Schätzle [KS1], [KS2], and [KS3]. See also [Sim].

iii) Observe that the Hodge decomposition (I.19) applied to the mean curvature vector $\vec{H}$ yields the system

$$
\left\{\begin{array}{l}
\Delta \vec{A}=\nabla \vec{H} \wedge \nabla^{\perp} \vec{n} \\
\Delta \vec{B}=-3 \nabla H \cdot \nabla^{\perp} \vec{n} .
\end{array}\right.
$$

Since $\int_{\Sigma}|\nabla \vec{A}|^{2}+|\nabla \vec{B}|^{2}=\int_{\Sigma} 4|\nabla H|^{2}+|H|^{2}|\nabla \vec{n}|^{2}$, optimal Wentetype estimates applied to (I.21) should produce interesting lower bounds for the Willmore energy of Willmore immersions of tori.

The paper is organized as follows. In Sect. 2, the conservation laws satisfied by Willmore surfaces (proof of Theorem I.1) are established. In Sect. 3, we give a proof of the regularity of weak Willmore immersions (Theorem I.3). The point-removability result for Willmore graphs (Theorem I.6) is proved in Sect. 4. Finally, the appendix is devoted to the study of various intrinsic properties of the Willmore operator $\mathscr{L}_{\vec{n}}$. 
Acknowledgements. The author wishes to express his gratitude to Robert Bryant for stimulating discussions about Willmore surfaces. The author also thanks E. Kuwert and R. Schätzle for their valuable comments and suggestions on the first draft of the present work. In particular, they drew attention on the existence in the literature (cf. $[\mathrm{Ru}]$ ) of a weak formulation of the Willmore Euler-Lagrange equation in dimension 3.

\section{Conservation laws for Willmore surfaces}

II.1 The Willmore Euler-Lagrange equation in divergence form. This subsection is devoted to proving Theorem I.1.

Throughout this section, the operators $\nabla, \nabla^{\perp}, d i v$, and $\Delta$ are understood with respect to the flat metric on the unit 2-dimensional disk $D^{2}=\{z \in \mathbb{C}$; $|z|<1$. Let $\vec{\Phi}$ be a smooth conformal embedding of the unit disk $D^{2}$ into $\mathbb{R}^{n}$, and let $\Sigma=\vec{\Phi}\left(D^{2}\right)$. We introduce the function $\lambda$ via

$$
e^{\lambda}=\left|\frac{\partial \vec{\Phi}}{\partial x_{1}}\right|=\left|\frac{\partial \vec{\Phi}}{\partial x_{2}}\right|
$$

where $x_{1}$ and $x_{2}$ are the real and imaginary parts of the coordinate $z$ on $D^{2}$. Because of the topology of $D^{2}$, the normal bundle to $\Sigma$ is trivial and there exists therefore a smooth map $\vec{n}(z)=\left(\vec{n}_{1}(z), \ldots, \vec{n}_{m-2}(z)\right)$ from $D^{2}$ into the orthonormal $(m-2)$-frames in $\mathbb{R}^{m}$ such that

$$
\left\{\vec{n}_{1}(z), \ldots, \vec{n}_{m-2}(z)\right\} \quad \text { is a positive orthonormal basis to } N_{\vec{\Phi}(z)} \Sigma,
$$

where $N_{\vec{\Phi}(z)} \Sigma=\left(T_{\vec{\Phi}(z)} \Sigma\right)^{\perp}$ is the $(m-2)$-plane orthonormal to the tangent plane $T_{\vec{\Phi}(z)} \Sigma$. We denote by $\left\{\vec{e}_{1}, \vec{e}_{2}\right\}$ the orthonormal basis of $T_{\vec{\Phi}(z)} \Sigma$ given by

$$
\vec{e}_{i}=e^{-\lambda} \frac{\partial \vec{\Phi}}{\partial x_{i}}
$$

With this notation, the second fundamental form $\mathbf{h}$, which is a symmetric 2-form on $T_{\vec{\Phi}(z)} \Sigma$ into $N_{\vec{\Phi}(z)} \Sigma$, may be expressed as

$$
\mathbf{h}=\sum_{\alpha, i, j} h_{i j}^{\alpha} \vec{n}_{\alpha}\left(\vec{e}_{i}\right)^{*} \otimes\left(\vec{e}_{j}\right)^{*}, \quad \text { with } h_{i j}^{\alpha}=-e^{-\lambda}\left(\frac{\partial \vec{n}_{\alpha}}{\partial x_{i}}, \vec{e}_{j}\right) .
$$

Hence the mean curvature vector $\vec{H}$ is

$$
\vec{H}=\sum_{\alpha=1}^{m-2} H^{\alpha} \vec{n}_{\alpha}=\frac{1}{2} \sum_{\alpha=1}^{m-2}\left(h_{11}^{\alpha}+h_{22}^{\alpha}\right) \vec{n}_{\alpha} .
$$

Let $\vec{n}$ be the $(m-2)$-vector of $\mathbb{R}^{m}$ defined as $\vec{n}=\vec{n}_{1} \wedge \cdots \wedge \vec{n}_{m-2}$. Using the operator $\star$, whose definiton was recalled in the Introduction, we may identify vectors and $(m-1)$-vectors in $\mathbb{R}^{m}$. For instance, there holds:

$$
\star\left(\vec{n} \wedge \vec{e}_{1}\right)=\vec{e}_{2} \quad \text { and } \quad \star\left(\vec{n} \wedge \vec{e}_{2}\right)=-\vec{e}_{1} .
$$


Since $\left\{\vec{e}_{1}, \vec{e}_{2}, \vec{n}_{1}, \ldots, \vec{n}_{m-2}\right\}$ forms a basis for $T_{\vec{\Phi}(z)} \mathbb{R}^{m}$, we have the expansion

$$
\nabla \vec{n}_{\alpha}=\sum_{\beta=1}^{m-2}\left(\nabla \vec{n}_{\alpha}, \vec{n}_{\beta}\right) \vec{n}_{\beta}+\sum_{i=1}^{2}\left(\nabla \vec{n}_{\alpha}, \vec{e}_{i}\right) \vec{e}_{i},
$$

valid for every $\alpha=1, \ldots, m-2$. Consequently, it follows that

$$
\star\left(\vec{n} \wedge \nabla^{\perp} \vec{n}_{\alpha}\right)=\left(\nabla^{\perp} \vec{n}_{\alpha}, \vec{e}_{1}\right) \vec{e}_{2}-\left(\nabla^{\perp} \vec{n}_{\alpha}, \vec{e}_{2}\right) \vec{e}_{1} .
$$

Note that the symmetry of the second fundamental form (i.e. $h_{i j}^{\alpha}=h_{j i}^{\alpha}$ ) and the conformality of $\vec{\Phi}$ imply

$$
\left(\frac{\partial \vec{n}_{\alpha}}{\partial x_{1}}, \vec{e}_{2}\right)=\left(\frac{\partial \vec{n}_{\alpha}}{\partial x_{2}}, \vec{e}_{1}\right) .
$$

Combining (II.4) and (II.5) thus yields

$$
\begin{aligned}
\star & \left(\vec{n} \wedge \nabla^{\perp} \vec{n}_{\alpha}\right) \\
= & \left(\begin{array}{c}
-\left(\frac{\partial \vec{n}_{\alpha}}{\partial x_{1}}, \vec{e}_{2}\right) \\
\left(\frac{\partial \vec{n}_{\alpha}}{\partial x_{1}}, \vec{e}_{1}\right)
\end{array}\right) \vec{e}_{2}+\left(\begin{array}{c}
\left(\frac{\partial \vec{n}_{\alpha}}{\partial x_{2}}, \vec{e}_{2}\right) \\
-\left(\frac{\partial \vec{n}_{\alpha}}{\partial x_{2}}, \vec{e}_{1}\right)
\end{array}\right) \vec{e}_{1} \\
= & \left(\begin{array}{l}
-\left(\frac{\partial \vec{n}_{\alpha}}{\partial x_{1}}, \vec{e}_{2}\right) \\
-\left(\frac{\partial \vec{n}_{\alpha}}{\partial x_{2}}, \vec{e}_{2}\right)
\end{array}\right) \vec{e}_{2}+\left(\begin{array}{c}
-\left(\frac{\partial \vec{n}_{\alpha}}{\partial x_{1}}, \vec{e}_{1}\right) \\
-\left(\frac{\partial \vec{n}_{\alpha}}{\partial x_{2}}, \vec{e}_{1}\right)
\end{array}\right) \vec{e}_{1} \\
& +\left[\left(\frac{\partial \vec{n}_{\alpha}}{\partial x_{1}}, \vec{e}_{1}\right)+\left(\frac{\partial \vec{n}_{\alpha}}{\partial x_{2}}, \vec{e}_{2}\right)\right]\left[\left(\begin{array}{l}
1 \\
0
\end{array}\right) \vec{e}_{1}+\left(\begin{array}{l}
0 \\
1
\end{array}\right) \vec{e}_{2}\right] .
\end{aligned}
$$

This implies

$$
\begin{aligned}
& \nabla \vec{n}_{\alpha}+\star\left(\vec{n} \wedge \nabla^{\perp} \vec{n}_{\alpha}\right) \\
& \quad=\sum_{\beta=1}^{m-2}\left(\nabla \vec{n}_{\alpha}, \vec{n}_{\beta}\right) \vec{n}_{\beta}-2 e^{\lambda} H^{\alpha}\left[\left(\begin{array}{l}
1 \\
0
\end{array}\right) \vec{e}_{1}+\left(\begin{array}{l}
0 \\
1
\end{array}\right) \vec{e}_{2}\right] .
\end{aligned}
$$

Substituting the identity $\partial_{x_{j}} \vec{\Phi}=e^{\lambda} \vec{e}_{j}$ into the latter, we reach the important equality

$$
\nabla \vec{n}_{\alpha}+\star\left(\vec{n} \wedge \nabla^{\perp} \vec{n}_{\alpha}\right)=\sum_{\beta=1}^{m-2}\left(\nabla \vec{n}_{\alpha}, \vec{n}_{\beta}\right) \vec{n}_{\beta}-2 H^{\alpha} \nabla \vec{\Phi} .
$$

Following the "Coulomb gauge extraction method" presented in the proof of Lemma 4.1.3 from [Hel], we may choose a trivialization $\left\{\vec{n}_{1}, \ldots, \vec{n}_{m-2}\right\}$ 
of the orthonormal frame bundle associated to our trivial bundle $N \Sigma$ and satisfying

$$
\operatorname{div}\left(\nabla \vec{n}_{\alpha}, \vec{n}_{\beta}\right)=0, \quad \forall 1 \leq \alpha, \beta \leq m-2 .
$$

The identities (II.8) and (II.9) may be combined so as to produce:

$$
\begin{aligned}
\operatorname{div} & \left(\nabla \vec{n}_{\alpha}+\star\left(\vec{n} \wedge \nabla^{\perp} \vec{n}_{\alpha}\right)\right) \\
= & \sum_{\beta=1}^{m-2}\left(\nabla \vec{n}_{\alpha}, \vec{n}_{\beta}\right) \cdot \nabla \vec{n}_{\beta}-2 \nabla H^{\alpha} \cdot \nabla \vec{\Phi}-2 H^{\alpha} \Delta \vec{\Phi} .
\end{aligned}
$$

On the other hand, an elementary classical computation gives

$$
\Delta \vec{\Phi}=2 e^{2 \lambda} \vec{H} .
$$

Bringing altogether the two last identities, we obtain now

$$
\begin{aligned}
\operatorname{div} & \left(\nabla \vec{n}_{\alpha}+\star\left(\vec{n} \wedge \nabla^{\perp} \vec{n}_{\alpha}\right)\right) \\
= & \sum_{\beta=1}^{m-2}\left(\nabla \vec{n}_{\alpha}, \vec{n}_{\beta}\right) \cdot \nabla \vec{n}_{\beta}-2 \nabla H^{\alpha} \cdot \nabla \vec{\Phi}-4 e^{2 \lambda} H^{\alpha} \vec{H} .
\end{aligned}
$$

Multiplying throughout by $H^{\alpha}$, summing over $\alpha=1, \ldots, m-2$, and using the expression for $H^{\alpha} \nabla \vec{\Phi}$ given in (II.8), we infer that

$$
\begin{aligned}
& \sum_{\alpha=1}^{m-2} H^{\alpha} \operatorname{div}\left(\nabla \vec{n}_{\alpha}+\star\left(\vec{n} \wedge \nabla^{\perp} \vec{n}_{\alpha}\right)\right)-\sum_{\alpha=1}^{m-2} \nabla H^{\alpha} \cdot \nabla \vec{n}_{\alpha} \\
& -\star \sum_{\alpha=1}^{m-2} \nabla H^{\alpha} \cdot \vec{n} \wedge \nabla^{\perp} \vec{n}_{\alpha} \\
& =\sum_{\alpha, \beta=1}^{m-2} H^{\alpha}\left(\nabla \vec{n}_{\alpha}, \vec{n}_{\beta}\right) \cdot \nabla \vec{n}_{\beta}-\sum_{\alpha, \beta=1}^{m-2} \nabla H^{\alpha} \cdot\left(\nabla \vec{n}_{\alpha}, \vec{n}_{\beta}\right) \vec{n}_{\beta} \\
& \quad-4 e^{2 \lambda} H^{\alpha} \vec{H} .
\end{aligned}
$$

For our future needs, we find helpful to recast (II.13) in the form

$$
\begin{aligned}
\sum_{\alpha=1}^{m-2} & H^{\alpha} \Delta \vec{n}_{\alpha}-\sum_{\alpha=1}^{m-2} \nabla H^{\alpha} \cdot \nabla \vec{n}_{\alpha}-\star \sum_{\alpha=1}^{m-2} \operatorname{div}\left(H^{\alpha} \vec{n} \wedge \nabla^{\perp} \vec{n}_{\alpha}\right) \\
= & \sum_{\alpha, \beta=1}^{m-2} H^{\alpha}\left(\nabla \vec{n}_{\alpha}, \vec{n}_{\beta}\right) \cdot \nabla \vec{n}_{\beta}-\sum_{\alpha, \beta=1}^{m-2} \nabla H^{\alpha} \cdot\left(\nabla \vec{n}_{\alpha}, \vec{n}_{\beta}\right) \vec{n}_{\beta} \\
& -2 \star \sum_{\alpha=1}^{m-2} H^{\alpha} \operatorname{div}\left(\vec{n} \wedge \nabla^{\perp} \vec{n}_{\alpha}\right)-4 e^{2 \lambda} H^{\alpha} \vec{H} .
\end{aligned}
$$


We next develop a more "tractable" expression for the second term in the right-hand-side of (II.14). To this end, we note first that (II.4) yields

$$
\begin{aligned}
\star & \operatorname{div}\left(\vec{n} \wedge \nabla^{\perp} \vec{n}_{\alpha}\right) \\
= & \left(\nabla^{\perp} \vec{n}_{\alpha}, \nabla \vec{e}_{1}\right) \vec{e}_{2}-\left(\nabla^{\perp} \vec{n}_{\alpha}, \nabla \vec{e}_{2}\right) \vec{e}_{1} \\
& +\left(\nabla^{\perp} \vec{n}_{\alpha}, \vec{e}_{1}\right) \cdot \nabla \vec{e}_{2}-\left(\nabla^{\perp} \vec{n}_{\alpha}, \vec{e}_{2}\right) \cdot \nabla \vec{e}_{1} \\
= & \sum_{\beta=1}^{m-2}\left(\nabla^{\perp} \vec{n}_{\alpha}, \vec{n}_{\beta}\right) \cdot\left[\left(\vec{n}_{\beta}, \nabla \vec{e}_{1}\right) \vec{e}_{2}-\left(\vec{n}_{\beta}, \nabla \vec{e}_{2}\right) \vec{e}_{1}\right] \\
& +\sum_{\beta=1}^{m-2}\left[\left(\nabla^{\perp} \vec{n}_{\alpha}, \vec{e}_{1}\right) \cdot\left(\nabla \vec{e}_{2}, \vec{n}_{\beta}\right)-\left(\nabla^{\perp} \vec{n}_{\alpha}, \vec{e}_{2}\right) \cdot\left(\nabla \vec{e}_{1}, \vec{n}_{\beta}\right)\right] \vec{n}_{\beta} .
\end{aligned}
$$

On the other hand, the symmetry of $\mathbf{h}$ implies that

$$
\begin{aligned}
\left(\nabla^{\perp} \vec{n}_{\alpha}, \vec{e}_{1}\right) \cdot\left(\nabla \vec{e}_{2}, \vec{n}_{\beta}\right)-\left(\nabla^{\perp} \vec{n}_{\alpha}, \vec{e}_{2}\right) \cdot\left(\nabla \vec{e}_{1}, \vec{n}_{\beta}\right) \\
=\left(-\frac{\partial \vec{n}_{\alpha}}{\partial x_{2}}, \vec{e}_{1}\right)\left(\frac{\partial \vec{e}_{2}}{\partial x_{1}}, \vec{n}_{\beta}\right)+\left(\frac{\partial \vec{n}_{\alpha}}{\partial x_{1}}, \vec{e}_{1}\right)\left(\frac{\partial \vec{e}_{2}}{\partial x_{2}}, \vec{n}_{\beta}\right) \\
+\left(\frac{\partial \vec{n}_{\alpha}}{\partial x_{2}}, \vec{e}_{2}\right)\left(\frac{\partial \vec{e}_{2}}{\partial x_{1}}, \vec{n}_{\beta}\right)-\left(\frac{\partial \vec{n}_{\alpha}}{\partial x_{1}}, \vec{e}_{2}\right)\left(\frac{\partial \vec{e}_{2}}{\partial x_{2}}, \vec{n}_{\beta}\right) \\
=\left(\frac{\partial \vec{n}_{\alpha}}{\partial x_{2}}, \vec{e}_{1}\right)\left(\frac{\partial \vec{n}_{\beta}}{\partial x_{1}}, \vec{e}_{2}\right)-\left(\frac{\partial \vec{n}_{\alpha}}{\partial x_{1}}, \vec{e}_{1}\right)\left(\frac{\partial \vec{n}_{\beta}}{\partial x_{2}}, \vec{e}_{2}\right) \\
-\left(\frac{\partial \vec{n}_{\alpha}}{\partial x_{2}}, \vec{e}_{2}\right)\left(\frac{\partial \vec{n}_{\beta}}{\partial x_{1}}, \vec{e}_{1}\right)+\left(\frac{\partial \vec{n}_{\alpha}}{\partial x_{1}}, \vec{e}_{2}\right)\left(\frac{\partial \vec{n}_{\beta}}{\partial x_{2}}, \vec{e}_{1}\right) \\
=e^{2 \lambda}\left[h_{21}^{\alpha} h_{12}^{\beta}-h_{11}^{\alpha} h_{22}^{\beta}-h_{22}^{\alpha} h_{11}^{\beta}+h_{12}^{\alpha} h_{21}^{\beta}\right] \\
=e^{2 \lambda} \sum_{i, j} h_{i j}^{\alpha} h_{i j}^{\beta}-4 e^{2 \lambda} H^{\alpha} H^{\beta} .
\end{aligned}
$$

Bringing altogether (II.15) and (II.16) produces:

$$
\begin{aligned}
& \star \sum_{\alpha=1}^{m-2} H^{\alpha} \operatorname{div}\left(\vec{n} \wedge \nabla^{\perp} \vec{n}_{\alpha}\right) \\
& =\sum_{\alpha, \beta=1}^{m-2} H^{\alpha}\left(\nabla^{\perp} \vec{n}_{\alpha}, \vec{n}_{\beta}\right) \cdot\left[\left(\vec{n}_{\beta}, \nabla \vec{e}_{1}\right) \vec{e}_{2}-\left(\vec{n}_{\beta}, \nabla \vec{e}_{2}\right) \vec{e}_{1}\right] \\
& \quad+e^{2 \lambda} \sum_{\alpha, \beta, i, j} h_{i j}^{\alpha} h_{i j}^{\beta}-4 e^{2 \lambda}|\vec{H}|^{2} \vec{H} .
\end{aligned}
$$


Again for our future purposes, we recast the latter in the form

$$
\begin{aligned}
-2 \star & \sum_{\alpha=1}^{m-2} H^{\alpha} \operatorname{div}\left(\vec{n} \wedge \nabla^{\perp} \vec{n}_{\alpha}\right)-4 e^{2 \lambda}|\vec{H}|^{2} \vec{H} \\
= & -2 \sum_{\alpha, \beta=1}^{m-2} H^{\alpha}\left(\nabla^{\perp} \vec{n}_{\alpha}, \vec{n}_{\beta}\right) \cdot\left[\left(\vec{n}_{\beta}, \nabla \vec{e}_{1}\right) \vec{e}_{2}-\left(\vec{n}_{\beta}, \nabla \vec{e}_{2}\right) \vec{e}_{1}\right] \\
& -2 e^{2 \lambda} \sum_{\alpha, \beta, i, j} h_{i j}^{\alpha} h_{i j}^{\beta}+4 e^{2 \lambda}|\vec{H}|^{2} \vec{H} .
\end{aligned}
$$

$\vec{H}$ is a section of $N \Sigma$. By definition, the covariant (negative) Laplacian of $\vec{H}$ relative to the connection derived from the orthogonal projection $\pi_{\vec{n}}$ (with respect to the standard scalar product in $\mathbb{R}^{m}$ ) on the fibers of $N \Sigma$ satisfies

$$
e^{2 \lambda} \Delta_{\perp} \vec{H}:=\pi_{\vec{n}} \operatorname{div}\left(\pi_{\vec{n}}(\nabla \vec{H})\right) .
$$

Introducing (II.9), the latter may be recast as

$$
\begin{aligned}
e^{2 \lambda} \Delta_{\perp} \vec{H}:= & \sum_{\alpha, \beta} \pi_{\vec{n}} \operatorname{div}\left(\nabla H^{\alpha} \vec{n}_{\alpha}+H^{\alpha}\left(\nabla \vec{n}_{\alpha}, \vec{n}_{\beta}\right) n_{\beta}\right) \\
= & \sum_{\alpha} \Delta H^{\alpha} \vec{n}_{\alpha}+2 \sum_{\alpha, \beta} \nabla H^{\alpha} \cdot\left(\nabla \vec{n}_{\alpha}, \vec{n}_{\beta}\right) \vec{n}_{\beta} \\
& +\sum_{\alpha, \beta, \gamma} H^{\alpha}\left(\nabla \vec{n}_{\alpha}, \vec{n}_{\beta}\right) \cdot\left(\nabla \vec{n}_{\beta}, \vec{n}_{\gamma}\right) \vec{n}_{\gamma} .
\end{aligned}
$$

Demanding that the embedding $\vec{\Phi}$ be Willmore is tantamount to assuming that (I.2) holds (see [Wei]), which in our notation becomes

$$
\Delta_{\perp} \vec{H}+\sum_{i, j, \alpha, \beta} h_{i j}^{\alpha} h_{i j}^{\beta} H^{\beta} \vec{n}_{\alpha}-2|\vec{H}|^{2} \vec{H}=0 .
$$

In this case, we deduce from (II.18) that

$$
\begin{aligned}
-2 \star & \sum_{\alpha=1}^{m-2} H^{\alpha} \operatorname{div}\left(\vec{n} \wedge \nabla^{\perp} \vec{n}_{\alpha}\right)-4 e^{2 \lambda}|\vec{H}|^{2} \vec{H} \\
= & -2 \sum_{\alpha, \beta=1}^{m-2} H^{\alpha}\left(\nabla^{\perp} \vec{n}_{\alpha}, \vec{n}_{\beta}\right) \cdot\left[\left(\vec{n}_{\beta}, \nabla \vec{e}_{1}\right) \vec{e}_{2}-\left(\vec{n}_{\beta}, \nabla \vec{e}_{2}\right) \vec{e}_{1}\right] \\
& +2 e^{2 \lambda} \Delta_{\perp} \vec{H} .
\end{aligned}
$$


Combining altogether (II.14), (II.19), and (II.21), we see that the conformal embedding $\vec{\Phi}$ is Willmore if and only if there holds:

$$
\begin{aligned}
& \sum_{\alpha=1}^{m-2} H^{\alpha} \Delta \vec{n}_{\alpha}-\sum_{\alpha=1}^{m-2} \nabla H^{\alpha} \cdot \nabla \vec{n}_{\alpha}-\star \sum_{\alpha=1}^{m-2} \operatorname{div}\left(H^{\alpha} \vec{n} \wedge \nabla^{\perp} \vec{n}_{\alpha}\right) \\
& =\sum_{\alpha, \beta=1}^{m-2} H^{\alpha}\left(\nabla \vec{n}_{\alpha}, \vec{n}_{\beta}\right) \cdot \nabla \vec{n}_{\beta}-\sum_{\alpha, \beta=1}^{m-2} \nabla H^{\alpha} \cdot\left(\nabla \vec{n}_{\alpha}, \vec{n}_{\beta}\right) \vec{n}_{\beta} \\
& \quad-2 \sum_{\alpha, \beta=1}^{m-2} H^{\alpha}\left(\nabla^{\perp} \vec{n}_{\alpha}, \vec{n}_{\beta}\right) \cdot\left[\left(\vec{n}_{\beta}, \nabla \vec{e}_{1}\right) \vec{e}_{2}-\left(\vec{n}_{\beta}, \nabla \vec{e}_{2}\right) \vec{e}_{1}\right] \\
& \quad+2 \sum_{\alpha} \Delta H^{\alpha} \vec{n}_{\alpha}+4 \sum_{\alpha, \beta} \nabla H^{\alpha} \cdot\left(\nabla \vec{n}_{\alpha}, \vec{n}_{\beta}\right) \vec{n}_{\beta} \\
& \quad+2 \sum_{\alpha, \beta, \gamma} H^{\alpha}\left(\nabla \vec{n}_{\alpha}, \vec{n}_{\beta}\right) \cdot\left(\nabla \vec{n}_{\beta}, \vec{n}_{\gamma}\right) \vec{n}_{\gamma} .
\end{aligned}
$$

Our next goal consists in showing that (II.22) can be brought into divergence form.

To this end, we first note that

$$
\begin{aligned}
&\left(\nabla^{\perp} \vec{n}_{\alpha}, \vec{n}_{\beta}\right) \cdot\left[\left(\vec{n}_{\beta}, \nabla \vec{e}_{1}\right) \vec{e}_{2}-\left(\vec{n}_{\beta}, \nabla \vec{e}_{2}\right) \vec{e}_{1}\right] \\
&=\left[\left(\frac{\partial \vec{n}_{\alpha}}{\partial x_{2}}, \vec{n}_{\beta}\right)\left(\frac{\partial \vec{n}_{\beta}}{\partial x_{1}}, \vec{e}_{1}\right)-\left(\frac{\partial \vec{n}_{\alpha}}{\partial x_{1}}, \vec{n}_{\beta}\right)\left(\frac{\partial \vec{n}_{\beta}}{\partial x_{2}}, \vec{e}_{1}\right)\right] \vec{e}_{2} \\
&+ {\left[\left(\frac{\partial \vec{n}_{\alpha}}{\partial x_{2}}, \vec{n}_{\beta}\right)\left(\frac{\partial \vec{n}_{\beta}}{\partial x_{1}}, \vec{e}_{2}\right)-\left(\frac{\partial \vec{n}_{\alpha}}{\partial x_{1}}, \vec{n}_{\beta}\right)\left(\frac{\partial \vec{n}_{\beta}}{\partial x_{2}}, \vec{e}_{2}\right)\right] \vec{e}_{1} } \\
&=-2\left(\frac{\partial \vec{n}_{\alpha}}{\partial x_{2}}, \vec{n}_{\beta}\right) e^{\lambda} H^{\beta}-\left(\frac{\partial \vec{n}_{\alpha}}{\partial x_{2}}, \vec{n}_{\beta}\right)\left(\frac{\partial \vec{n}_{\beta}}{\partial x_{2}}, \vec{e}_{2}\right) \\
&\left.-\left(\frac{\partial \vec{n}_{\alpha}}{\partial x_{1}}, \vec{n}_{\beta}\right)\left(\frac{\partial \vec{n}_{\beta}}{\partial x_{1}}, \vec{e}_{2}\right)\right] \vec{e}_{2} \\
&+\left[-2\left(\frac{\partial \vec{n}_{\alpha}}{\partial x_{1}}, \vec{n}_{\beta}\right) e^{\lambda} H^{\beta}-\left(\frac{\partial \vec{n}_{\alpha}}{\partial x_{1}}, \vec{n}_{\beta}\right)\left(\frac{\partial \vec{n}_{\beta}}{\partial x_{1}}, \vec{e}_{1}\right)\right. \\
&\left.\quad-\left(\frac{\partial \vec{n}_{\alpha}}{\partial x_{2}}, \vec{n}_{\beta}\right)\left(\frac{\partial \vec{n}_{\beta}}{\partial x_{2}}, \vec{e}_{1}\right)\right] \vec{e}_{1} \\
&=-2 H^{\beta}\left(\nabla \vec{n}_{\alpha}, \vec{n}_{\beta}\right) \cdot \nabla \vec{\Phi} \\
&-\left(\nabla \vec{n}_{\alpha}, \vec{n}_{\beta}\right) \cdot\left[\left(\nabla \vec{n}_{\beta}, \vec{e}_{2}\right) \vec{e}_{2}+\left(\nabla \vec{n}_{\beta}, \vec{e}_{1}\right) \vec{e}_{1}\right] .
\end{aligned}
$$


Whence we have

$$
\begin{aligned}
& -2 \sum_{\alpha, \beta=1}^{m-2} H^{\alpha}\left(\nabla^{\perp} \vec{n}_{\alpha}, \vec{n}_{\beta}\right) \cdot\left[\left(\vec{n}_{\beta}, \nabla \vec{e}_{1}\right) \vec{e}_{2}-\left(\vec{n}_{\beta}, \nabla \vec{e}_{2}\right) \vec{e}_{1}\right] \\
& =4 \sum_{\alpha=1}^{m-2} H^{\alpha} H^{\beta}\left(\nabla \vec{n}_{\alpha}, \vec{n}_{\beta}\right) \cdot \nabla \vec{\Phi} \\
& \quad+2 \sum_{\alpha, \beta=1}^{m-2} H^{\alpha}\left(\nabla \vec{n}_{\alpha}, \vec{n}_{\beta}\right) \cdot\left[\left(\nabla \vec{n}_{\beta}, \vec{e}_{2}\right) \vec{e}_{2}+\left(\nabla \vec{n}_{\beta}, \vec{e}_{1}\right) \vec{e}_{1}\right] .
\end{aligned}
$$

Next, it follows from $\left(\nabla \vec{n}_{\alpha}, \vec{n}_{\beta}\right)=-\left(\nabla \vec{n}_{\beta}, \vec{n}_{\alpha}\right)$ that

$$
\sum_{\alpha, \beta=1}^{m-2} H^{\alpha} H^{\beta}\left(\nabla \vec{n}_{\alpha}, \vec{n}_{\beta}\right) \cdot \nabla \vec{\Phi} \equiv 0
$$

which is introduced into (II.24) to yield

$$
\begin{aligned}
& -2 \sum_{\alpha, \beta=1}^{m-2} H^{\alpha}\left(\nabla^{\perp} \vec{n}_{\alpha}, \vec{n}_{\beta}\right) \cdot\left[\left(\vec{n}_{\beta}, \nabla \vec{e}_{1}\right) \vec{e}_{2}-\left(\vec{n}_{\beta}, \nabla \vec{e}_{2}\right) \vec{e}_{1}\right] \\
& \quad+2 \sum_{\alpha, \beta, \gamma} H^{\alpha}\left(\nabla \vec{n}_{\alpha}, \vec{n}_{\beta}\right) \cdot\left(\nabla \vec{n}_{\beta}, \vec{n}_{\gamma}\right) \vec{n}_{\gamma} \\
& =2 \sum_{\alpha, \beta=1}^{m-2} H^{\alpha}\left(\nabla \vec{n}_{\alpha}, \vec{n}_{\beta}\right) \cdot \nabla \vec{n}_{\beta} .
\end{aligned}
$$

Upon substituting the latter into (II.22), we conclude that the conformal embedding $\vec{\Phi}$ is Willmore if and only if

$$
\begin{aligned}
& \sum_{\alpha=1}^{m-2} H^{\alpha} \Delta \vec{n}_{\alpha}-\sum_{\alpha=1}^{m-2} \nabla H^{\alpha} \cdot \nabla \vec{n}_{\alpha}-\star \sum_{\alpha=1}^{m-2} \operatorname{div}\left(H^{\alpha} \vec{n} \wedge \nabla^{\perp} \vec{n}_{\alpha}\right) \\
& =3 \sum_{\alpha, \beta=1}^{m-2} H^{\alpha}\left(\nabla \vec{n}_{\alpha}, \vec{n}_{\beta}\right) \cdot \nabla \vec{n}_{\beta}+2 \sum_{\alpha=1}^{m-2} \Delta H^{\alpha} \vec{n}_{\alpha} \\
& \quad+3 \sum_{\alpha, \beta=1}^{m-2} \nabla H^{\alpha} \cdot\left(\nabla \vec{n}_{\alpha}, \vec{n}_{\beta}\right) \vec{n}_{\beta} .
\end{aligned}
$$


With the help of the condition (II.9), we recast (II.27) in the form

$$
\begin{gathered}
\sum_{\alpha=1}^{m-2} H^{\alpha} \Delta \vec{n}_{\alpha}-\sum_{\alpha=1}^{m-2} \nabla H^{\alpha} \cdot \nabla \vec{n}_{\alpha}-\star \sum_{\alpha=1}^{m-2} \operatorname{div}\left(H^{\alpha} \vec{n} \wedge \nabla^{\perp} \vec{n}_{\alpha}\right) \\
=3 \operatorname{div}\left[\sum_{\alpha, \beta=1}^{m-2} H^{\alpha}\left(\nabla \vec{n}_{\alpha}, \vec{n}_{\beta}\right) \vec{n}_{\beta}\right]+2 \sum_{\alpha=1}^{m-2} \Delta H^{\alpha} \vec{n}_{\alpha} .
\end{gathered}
$$

Let us note that

$$
-\star \operatorname{div} \sum_{\alpha=1}^{m-2} H^{\alpha} \vec{n} \wedge \nabla^{\perp} \vec{n}_{\alpha}=\operatorname{div} \star\left(\nabla^{\perp} \vec{n} \wedge \vec{H}\right),
$$

and that

$$
\sum_{\alpha, \beta=1}^{m-2} H^{\alpha}\left(\nabla \vec{n}_{\alpha}, \vec{n}_{\beta}\right) \vec{n}_{\beta}=\pi_{\vec{n}}(\nabla \vec{H})-\sum_{\alpha=1}^{m-2} \nabla H^{\alpha} \vec{n}_{\alpha} .
$$

These last two identities enable us to finally express the Willmore equation (II.28) in the desired divergence form; namely:

$$
\Delta \vec{H}-3 \operatorname{div}\left(\pi_{\vec{n}}(\nabla \vec{H})\right)+\operatorname{div} \star\left(\nabla^{\perp} \vec{n} \wedge \vec{H}\right)=0 .
$$

II.2 The proofs of the conservation laws (I.11) and (I.12), and of the identity (I.13). Assume that (I.9) holds on $D^{2}$ for a bilipschitz conformal immersion $\vec{\Phi}$. Consider the map $\vec{L}$ from $D^{2}$ into $\mathbb{R}^{m}$ defined via

$$
\nabla^{\perp} \vec{L}:=\nabla \vec{H}-3 \pi_{\vec{n}}(\nabla \vec{H})+\star\left(\nabla^{\perp} \vec{n} \wedge \vec{H}\right) .
$$

A simple argument guarantees that $\vec{L}$ is uniquely defined up to a multiplicative constant.

Our first aim is to establish (I.11). Using again a local framing of the normal and of the tangent bundles to the immersion, we have:

$$
\begin{aligned}
\star\left(\nabla^{\perp} \vec{n} \wedge \vec{H}\right) & =\star \sum_{\beta, \gamma=1}^{m-2} H^{\gamma} \wedge_{\alpha<\beta} \vec{n}_{\alpha} \wedge \nabla^{\perp} \vec{n}_{\beta} \wedge_{\beta>\alpha} \vec{n}_{\alpha} \wedge \vec{n}_{\gamma} \\
& =-\star \sum_{\gamma=1}^{m-2} H^{\gamma} \vec{n} \wedge \nabla^{\perp} \vec{n}_{\gamma} \\
& =-\sum_{\gamma=1}^{m-2} H^{\gamma}\left[\left(\nabla^{\perp} \vec{n}_{\gamma}, \vec{e}_{1}\right) \vec{e}_{2}-\left(\nabla^{\perp} \vec{n}_{\gamma}, \vec{e}_{2}\right) \vec{e}_{1}\right] .
\end{aligned}
$$


Accordingly, we find

$$
\star\left(\nabla^{\perp} \vec{n} \wedge \vec{H}\right) \cdot \nabla \vec{\Phi}=-\sum_{\gamma=1}^{m-2} H^{\gamma} \nabla \vec{n}_{\gamma} \cdot \nabla \vec{\Phi} .
$$

Since clearly $\pi_{\vec{n}}(\nabla \vec{H}) \cdot \nabla \vec{\Phi}=0$, we have thus proved that

$$
\nabla^{\perp} \vec{L} \cdot \nabla \vec{\Phi}=\nabla \vec{H} \cdot \nabla \vec{\Phi}-\sum_{\gamma=1}^{m-2} H^{\gamma} \nabla \vec{n}_{\gamma} \cdot \nabla \vec{\Phi} .
$$

On the other hand, the fact that $\vec{n}_{\gamma} \cdot \nabla \vec{\Phi}=0$ implies:

$$
\nabla \vec{H} \cdot \nabla \vec{\Phi}=\sum_{\gamma=1}^{m-2} H^{\gamma} \nabla \vec{n}_{\gamma} \cdot \nabla \vec{\Phi} .
$$

The desired identity (I.11) follows at once from substituting (II.36) into (II.35).

Our second aim consists in recovering identity (I.12). To this end, we first note that

$$
\nabla \vec{\Phi} \wedge \nabla \vec{H}=\sum_{\gamma=1}^{m-2} \nabla H^{\gamma} \cdot \nabla \vec{\Phi} \wedge \vec{n}_{\gamma}+\sum_{\gamma=1}^{m-2} H^{\gamma} \nabla \vec{\Phi} \wedge \nabla \vec{n}_{\gamma} .
$$

Secondly, there holds

$$
\begin{aligned}
-3 & \nabla \vec{\Phi} \wedge \pi_{\vec{n}}(\nabla \vec{H}) \\
& =-3 \nabla \vec{\Phi} \wedge \pi_{\vec{n}}\left(\sum_{\gamma=1}^{m-2} \nabla H^{\gamma} \cdot \vec{n}_{\gamma}\right)-3 \nabla \vec{\Phi} \wedge \pi_{\vec{n}}\left(\sum_{\gamma=1}^{m-2} H^{\gamma} \nabla \vec{n}_{\gamma}\right) \\
& =-3 \nabla \vec{\Phi} \wedge \sum_{\gamma=1}^{m-2} \nabla H^{\gamma} \cdot \vec{n}_{\gamma}-3 \nabla \vec{\Phi} \wedge \sum_{\alpha, \gamma=1}^{m-2} H^{\gamma}\left(\nabla \vec{n}_{\gamma}, \vec{n}_{\alpha}\right) \vec{n}_{\alpha} .
\end{aligned}
$$

And thirdly, using (II.34), we find

$$
\begin{aligned}
\nabla \vec{\Phi} \wedge \star\left(\nabla^{\perp} \vec{n} \wedge \vec{H}\right) & =-\nabla \vec{\Phi} \wedge \sum_{\gamma=1}^{m-2} H^{\gamma}\left[\left(\nabla^{\perp} \vec{n}_{\gamma}, \vec{e}_{1}\right) \vec{e}_{2}-\left(\nabla^{\perp} \vec{n}_{\gamma}, \vec{e}_{2}\right) \vec{e}_{1}\right] \\
& =\sum_{\gamma=1}^{m-2} H^{\gamma}\left[\left(\partial_{x_{2}} n_{\gamma}, \partial_{x_{1}} \vec{\Phi}\right)-\left(\partial_{x_{1}} n_{\gamma}, \partial_{x_{2}} \vec{\Phi}\right)\right] \vec{e}_{1} \wedge \vec{e}_{2}=0
\end{aligned}
$$

owing to the symmetry of the second fundamental form. 
Bringing altogether (II.37), (II.38), and (II.39) finally yields the desired

$$
\begin{aligned}
\nabla \vec{\Phi} \wedge \nabla^{\perp} \vec{L}= & -2 \sum_{\gamma=1}^{m-2} \nabla H^{\gamma} \cdot \nabla \vec{\Phi} \wedge \vec{n}_{\gamma}+\sum_{\gamma=1}^{m-2} H^{\gamma} \nabla \vec{\Phi} \wedge \nabla \vec{n}_{\gamma} \\
& -3 \nabla \vec{\Phi} \wedge \sum_{\alpha, \gamma=1}^{m-2} H^{\gamma}\left(\nabla \vec{n}_{\gamma}, \vec{n}_{\alpha}\right) \vec{n}_{\alpha} \\
= & -2 \sum_{\gamma=1}^{m-2} \nabla H^{\gamma} \cdot \nabla \vec{\Phi} \wedge \vec{n}_{\gamma}-2 \sum_{\gamma=1}^{m-2} H^{\gamma} \nabla \vec{\Phi} \wedge \nabla \vec{n}_{\gamma} \\
= & \nabla \vec{\Phi} \wedge \nabla \vec{H} .
\end{aligned}
$$

We shall now compute the term ${ }^{4} \nabla\left(\star(\vec{n}\llcorner\vec{H}))\left\llcorner\nabla^{\perp} \vec{\Phi}\right.\right.$. For this, we first observe that

$$
\vec{n}\left\llcorner\vec{H}=\sum_{\gamma=1}^{m-2}(-1)^{\gamma-1} H^{\gamma} \wedge_{\alpha \neq \gamma} \vec{n}_{\alpha} .\right.
$$

Hence there holds

$$
\star\left(\vec{n}\llcorner\vec{H})=(-1)^{m-3} \vec{e}_{1} \wedge \vec{e}_{2} \wedge \vec{H},\right.
$$

thereby yielding:

$$
(-1)^{m-3} \nabla\left[\star(\vec{n}\llcorner\vec{H})]=\vec{e}_{1} \wedge \vec{e}_{2} \nabla \vec{H}+\nabla \vec{e}_{1} \wedge \vec{e}_{2} \wedge \vec{H}+\vec{e}_{1} \wedge \nabla \vec{e}_{2} \wedge \vec{H} .\right.
$$

Next, we have that

$$
\begin{aligned}
\left(\vec{e}_{1} \wedge \vec{e}_{2} \wedge \nabla \vec{H}\right)\left\llcorner\nabla^{\perp} \vec{\Phi}=\right. & e^{\lambda} \vec{e}_{2} \wedge \frac{\partial \vec{H}}{\partial x_{2}}+e^{\lambda}\left(\frac{\partial \vec{n}_{\gamma}}{\partial x_{2}}, \vec{e}_{1}\right) \vec{e}_{1} \wedge \vec{e}_{2} \\
& +e^{\lambda} \vec{e}_{1} \wedge \frac{\partial \vec{H}}{\partial x_{1}}-e^{\lambda}\left(\frac{\partial \vec{n}_{\gamma}}{\partial x_{1}}, \vec{e}_{2}\right) \vec{e}_{1} \wedge \vec{e}_{2}
\end{aligned}
$$

This last identity may further be simplified using the symmetry of the second fundamental form. More precisely, (II.44) becomes:

$$
\begin{aligned}
\left(\vec{e}_{1} \wedge \vec{e}_{2} \wedge \nabla \vec{H}\right)\left\llcorner\nabla^{\perp} \vec{\Phi}\right. & =e^{\lambda} \vec{e}_{2} \wedge \frac{\partial \vec{H}}{\partial x_{2}}+e^{\lambda} \vec{e}_{1} \wedge \frac{\partial \vec{H}}{\partial x_{1}} \\
& =\nabla \vec{\Phi} \wedge \nabla \vec{H} .
\end{aligned}
$$

${ }^{4}$ The reader will find a rationale for looking at this particular term by considering the case $m=3$, whereby all of the necessary computations greatly simplify. 
On the other hand, there holds

$$
\begin{aligned}
\left(\nabla \vec{e}_{1} \wedge \vec{e}_{2} \wedge \vec{H}\right)\left\llcorner\nabla^{\perp} \vec{\Phi}=\right. & -\left(\frac{\vec{e}_{1}}{\partial x_{1}} \wedge \vec{e}_{2} \wedge \vec{H}\right)\left\llcorner\frac{\partial \vec{\Phi}}{\partial x_{2}}\right. \\
& +\left(\frac{\vec{e}_{1}}{\partial x_{2}} \wedge \vec{e}_{2} \wedge \vec{H}\right)\left\llcorner\frac{\partial \vec{\Phi}}{\partial x_{1}}\right. \\
= & -e^{\lambda}\left(\frac{\partial \vec{e}_{1}}{\partial x_{1}}, \vec{e}_{2}\right) \vec{e}_{2} \wedge \vec{H}+e^{\lambda} \frac{\partial \vec{e}_{1}}{\partial x_{1}} \wedge \vec{H} \\
= & \sum_{\gamma=1}^{m-2} e^{\lambda}\left(\frac{\partial \vec{e}_{1}}{\partial x_{1}}, \vec{n}_{\gamma}\right) \vec{n}_{\gamma} \wedge \vec{H},
\end{aligned}
$$

and similarly

$$
\begin{aligned}
\left(\vec{e}_{1} \wedge \nabla \vec{e}_{2} \wedge \vec{H}\right)\left\llcorner\nabla^{\perp} \vec{\Phi}=\right. & -\left(\vec{e}_{1} \wedge \frac{\vec{e}_{2}}{\partial x_{1}} \wedge \vec{H}\right)\left\llcorner\frac{\partial \vec{\Phi}}{\partial x_{2}}\right. \\
& +\left(\vec{e}_{1} \wedge \frac{\vec{e}_{2}}{\partial x_{2}} \wedge \vec{H}\right)\left\llcorner\frac{\partial \vec{\Phi}}{\partial x_{1}}\right. \\
= & -e^{\lambda}\left(\frac{\partial \vec{e}_{2}}{\partial x_{1}}, \vec{e}_{1}\right) \vec{e}_{1} \wedge \vec{H}+e^{\lambda} \frac{\partial \vec{e}_{2}}{\partial x_{2}} \wedge \vec{H} \\
= & \sum_{\gamma=1}^{m-2} e^{\lambda}\left(\frac{\partial \vec{e}_{2}}{\partial x_{2}}, \vec{n}_{\gamma}\right) \vec{n}_{\gamma} \wedge \vec{H} .
\end{aligned}
$$

Adding (II.46) and (II.47) together, we find that

$$
\begin{aligned}
& \left(\nabla \vec{e}_{1} \wedge \vec{e}_{2} \wedge \vec{H}\right)\left\llcorner\nabla^{\perp} \vec{\Phi}+\left(\vec{e}_{1} \wedge \nabla \vec{e}_{2} \wedge \vec{H}\right)\left\llcorner\nabla^{\perp} \vec{\Phi}\right.\right. \\
& =\sum_{\gamma=1}^{m-2} e^{\lambda}\left[\left(\frac{\partial \vec{e}_{1}}{\partial x_{1}}, \vec{n}_{\gamma}\right)+\left(\frac{\partial \vec{e}_{2}}{\partial x_{2}}, \vec{n}_{\gamma}\right)\right] \vec{n}_{\gamma} \wedge \vec{H} \\
& =2 e^{2 \lambda} \vec{H} \wedge \vec{H}=0 .
\end{aligned}
$$

We may now combine (II.43), (II.45), and (II.48) to deduce the identity

$$
(-1)^{m-3} \nabla\left[\star ( \vec { n } \llcorner \vec { H } ) ] \left\llcorner\nabla^{\perp} \vec{\Phi}=\nabla \vec{\Phi} \wedge \nabla \vec{H} .\right.\right.
$$

Upon introducing (II.40) into the latter, we have proved that

$$
\nabla \vec{\Phi} \wedge \nabla^{\perp} \vec{L}=2(-1)^{m-2} \nabla\left[\star ( \vec { n } \llcorner \vec { H } ) ] \left\llcorner\nabla^{\perp} \vec{\Phi} .\right.\right.
$$

This is the desired result, thereby ending the proof of the conservation law (I.12). 
In order to complete the proof of Theorem I.4, there remains to establish identity (I.13). To this end, we first note that

$$
\star\left(\vec{n}\llcorner\vec{H})=\star\left(\sum_{\gamma=1}^{m-2}(-1)^{\gamma-1} H^{\gamma} \wedge_{\alpha \neq \gamma} \vec{n}_{\alpha}\right)=(-1)^{m-3} \vec{e}_{1} \wedge \vec{e}_{2} \wedge \vec{H}\right.
$$

From this, it follows at once that

$$
\star\left(\vec { n } \llcorner \vec { H } ) \left\llcorner\nabla \vec{\Phi}=(-1)^{m-2} \nabla^{\perp} \vec{\Phi} \wedge \vec{H} .\right.\right.
$$

Hence, from the defining identity for $R$, namely

$$
\nabla \vec{R}:=\nabla \vec{\Phi} \wedge \vec{L}+2(-1)^{m}[\star(\vec{n}\llcorner\vec{H})] \wedge \nabla \vec{\Phi},
$$

we find

$$
\nabla \vec{R}=\nabla \vec{\Phi} \wedge \vec{L}+2 \nabla^{\perp} \vec{\Phi} \wedge \vec{H}
$$

Using $\vec{n}\llcorner\nabla \vec{\Phi}=0$, we infer from (II.54) that

$$
\vec{n} \bullet \nabla^{\perp} \vec{R}=-\left(\vec{n}\llcorner\vec{L}) \wedge \nabla^{\perp} \vec{\Phi}+2(\vec{n}\llcorner\vec{H}) \wedge \nabla \vec{\Phi} .\right.
$$

Let us next introduce the decomposition $\vec{L}=\vec{N}+\vec{T}$, where $\vec{N}=\pi_{\vec{n}}(\vec{L})$ is normal to $T_{\vec{\Phi}(x)} \vec{\Phi}\left(D^{2}\right)$, while $\vec{T}$ is contained in $T_{\vec{\Phi}(x)} \vec{\Phi}\left(D^{2}\right)$. In particular, we thus have $\vec{n}\llcorner\vec{L}=\vec{n}\llcorner\vec{N}$. Furthermore, there holds:

$$
\begin{aligned}
\star\left[\left(\vec{n}\llcorner\vec{N}) \wedge \nabla^{\perp} \vec{\Phi}\right]\right. & =\star\left[\sum_{\gamma=1}^{m-2}(-1)^{\gamma-1} N_{\gamma} \wedge_{\alpha \neq \gamma} \vec{n}_{\alpha} \wedge \nabla^{\perp} \vec{\Phi}\right] \\
& =(-1)^{m-1} \nabla \vec{\Phi} \wedge \vec{N} .
\end{aligned}
$$

A similar computation shows that

$$
\star\left[(\vec{n}\llcorner\vec{H}) \wedge \nabla \vec{\Phi}]=(-1)^{m} \nabla^{\perp} \vec{\Phi} \wedge \vec{H} .\right.
$$

Combining (II.55), (II.56), and (II.57) produces now:

$$
\begin{aligned}
(-1)^{m} \star\left[\vec{n} \bullet \nabla^{\perp} \vec{R}\right] & =\nabla \vec{\Phi} \wedge \vec{N}+2 \nabla^{\perp} \vec{\Phi} \wedge \vec{H} \\
& =\nabla \vec{R}-\nabla \vec{\Phi} \wedge \vec{T} .
\end{aligned}
$$

We consider next the function $S$ on $D^{2}$ defined through the identity $\nabla S=\vec{L} \cdot \nabla \vec{\Phi}$. Since $\vec{T}=\vec{L} \cdot \vec{e}_{1} \vec{e}_{1}+\vec{L} \cdot \vec{e}_{2} \vec{e}_{2}$, we derive easily

$$
e^{\lambda} \vec{T}=\frac{\partial S}{\partial x_{1}} \vec{e}_{1}+\frac{\partial S}{\partial x_{2}} \vec{e}_{2} .
$$

This implies that

$$
\nabla \vec{\Phi} \wedge \vec{T}=-\vec{e}_{1} \wedge \vec{e}_{2} \nabla^{\perp} S=-\star \vec{n} \nabla^{\perp} S .
$$

Finally, bringing altogether (II.58) and (II.60), we recover (I.13), thereby completing the proof of Theorem I.4. 


\section{The regularity of weak Willmore graphs}

This section is devoted to the proof of the regularity result stated in Theorem I.5.

With the help of the results derived in the previous section, we shall first demonstrate that the second derivative of the conformal immersion $\vec{\Phi}$ belongs to the Lorentz space ${ }^{5} L^{2,1}$; namely:

$$
\int_{0}^{+\infty}\left|\left\{x \in \Sigma:\left|\nabla^{2} \vec{\Phi}\right|(x) \geq \mu\right\}\right|^{\frac{1}{2}} d \mu<+\infty .
$$

The fact that $\nabla^{2} \vec{\Phi}$ lies in $L^{2,1}$ implies in particular that the gradient of the Gauss map $\vec{n}$ also belongs to $L^{2,1}$. A generalized version of the Sobolev embedding theorem then shows that the Gauss map is continuous. The immersion thus looks locally like a graph. Once this is established, we shall have the means to obtain the $\epsilon$-regularity result stated in Theorem I.5.

III.1 Proof of the fact that $\nabla^{2} \vec{\Phi}$ lies in $L^{2,1}$. We begin by observing that the gradient of $\vec{L}$ belongs to $L^{1} \oplus H^{-1}$. From this, it follows that $\vec{L}$ is in the space $L^{2, \infty}$ (which is the Marcinkiewicz space $L^{2}$-weak, see [Ta2]). Thus, from (II.53), we deduce that $\nabla \vec{R} \in L^{2, \infty}$.

For the reader's convenience, we recall a Wente-type estimate established in [Hel, cf. Theorem 3.4.5]. Let $a$ and $b$ be two functions on $D^{2}$ such that $\nabla a \in L^{2, \infty}\left(D^{2}\right)$ and $\nabla b \in L^{2}\left(D^{2}\right)$. Then there is a unique solution $\varphi$ in $W_{0}^{1,2}\left(D^{2}, \mathbb{R}\right)$ satisfying

$$
\begin{cases}\Delta \varphi=\nabla a \cdot \nabla^{\perp} b & \text { in } D^{2} \\ \varphi=0 & \text { on } \partial D^{2} .\end{cases}
$$

Moreover, there exists a positive constant $C$, independent of $a$ and $b$, such that

$$
\|\nabla \varphi\|_{L^{2}\left(D^{2}\right)} \leq C\|\nabla a\|_{L^{2, \infty}\left(D^{2}\right)}\|\nabla b\|_{L^{2}\left(D^{2}\right)} .
$$

This result may be directly applied to (I.14) so as to conclude that $\nabla \vec{R} \in L^{2}$. With the help of (I.13), we find similarly that $\nabla S \in L^{2}$.

The celebrated compensation compactness result by Coifman, Lions, Meyer, and Semmes [CLMS] (see also Theorems 3.2.2 and 3.2.9 in [Hel]) may also be applied to (I.14). It shows that $\nabla^{2} \vec{R} \in L^{1}$. Scalar-multiplying (I.13) throughout by $\star \vec{n}$ enables us to conclude that $\nabla S \in W^{1,1}$, which follows from $\nabla^{2} \vec{R} \in L^{1}$ and $\vec{n} \in W^{1,2}$.

Let us note next that (II.54) gives:

$$
\frac{\partial \vec{R}}{\partial x_{1}}=\frac{\partial \vec{\Phi}}{\partial x_{1}} \wedge \vec{L}-2 \frac{\partial \vec{\Phi}}{\partial x_{2}} \wedge \vec{H}
$$

5 An introduction to Lorentz spaces may be found in [Ta2]. 
Applying the interior product with $\vec{e}_{2}$ to this identity yields that

$$
-2 e^{\lambda} \vec{H}=\frac{\partial \vec{R}}{\partial x_{1}}\left\llcorner\vec{e}_{2}+\left(\vec{L} \cdot \vec{e}_{2}\right) \frac{\partial \vec{\Phi}}{\partial x_{1}}=\frac{\partial \vec{R}}{\partial x_{1}}\left\llcorner\vec{e}_{2}+\frac{\partial S}{\partial x_{2}} \vec{e}_{1} .\right.\right.
$$

By introducing our aforementioned findings, there follows that $\vec{H} \in W^{1,1}$, and thus, in particular, that $\vec{H} \in L^{2,1}$. Using now (II.11) and calling upon Theorem 3.3.3 from [Hel], we conclude at once that $\nabla^{2} \vec{\Phi}$ belongs to $L^{2,1}$, as desired.

III.2 Proof of Theorem I.5. Suppose that $\vec{\Phi}$ is a conformal Willmore immersion with second derivative in the Lorentz space $L^{2,1}$. We shall establish the $\epsilon$-regularity result stated in Theorem I.5.

According to Definition I.2, assuming that $\vec{\Phi}$ is a weak Willmore immersion is tantamount to requiring that the mean curvature $\vec{H}$ satisfy (I.9). Let us denote by $\chi$ a smooth cut-off function equal to 1 on $D_{1 / 2}^{2}$ (the two-dimensional disk of radius $1 / 2$ centered on the origin) and compactly supported in $D^{2}$. Since $\mathcal{L}_{\vec{n}} \vec{H}=0$, we have

$$
\begin{aligned}
\mathcal{L}_{\vec{n}}(\chi \vec{H})= & 2 \operatorname{div}(\nabla \chi \vec{H})-\vec{H} \Delta \chi-6 \operatorname{div}\left(\pi_{\vec{n}}(\vec{H}) \nabla \chi\right) \\
& +3(\vec{H} \cdot \nabla \vec{n}) \cdot \vec{n} \cdot \nabla \chi+3(\vec{H} \cdot \vec{n}) \cdot \nabla \vec{n} \cdot \nabla \chi \\
& -3 \Delta \chi \pi_{\vec{n}}(\vec{H})-\vec{H} \wedge \nabla^{\perp} \vec{n} \cdot \nabla \chi .
\end{aligned}
$$

Let now

$$
\vec{g}_{1}=2 \operatorname{div}(\nabla \chi \vec{H})-\vec{H} \Delta \chi-6 \operatorname{div}\left(\pi_{\vec{n}}(\vec{H}) \nabla \chi\right)-3 \Delta \chi \pi_{\vec{n}}(\vec{H}),
$$

and

$$
\vec{g}_{2}=3(\vec{H} \cdot \nabla \vec{n}) \cdot \vec{n} \nabla \chi+3(\vec{H} \cdot \vec{n}) \cdot \nabla \vec{n} \cdot \nabla \chi-\vec{H} \wedge \nabla^{\perp} \vec{n} \cdot \nabla \chi .
$$

Observe that there holds

$$
\left\|\vec{g}_{1}\right\|_{H^{-1}\left(D^{2}\right)}^{2} \leq C \int_{D^{2} \backslash D_{1 / 2}^{2}}|\vec{H}|^{2},
$$

and

$$
\left\|\vec{g}_{2}\right\|_{L^{1}\left(D^{2}\right)} \leq C \int_{D^{2} \backslash D_{1 / 2}^{2}}|\vec{H}||\nabla \vec{n}| .
$$

Let $\vec{v}_{1}$ be the solution of (A.5) given by Lemma A.1 with $\vec{g}=\vec{g}_{1}$; and let $\vec{v}_{2}$ be the solution of (A.30) given by Lemma A.3 with $\vec{g}=\vec{g}_{2}$ (refer to the appendix). These solutions satisfy the estimates

$$
\left\|\nabla \vec{v}_{1}\right\|_{L^{2}} \leq C\left[\int_{D^{2} \backslash D_{1 / 2}^{2}}|\vec{H}|^{2}\right]^{\frac{1}{2}},
$$


and

$$
\left\|\nabla \vec{v}_{2}\right\|_{L^{2, \infty}} \leq C \int_{D^{2} \backslash D_{1 / 2}^{2}}|\vec{H}||\nabla \vec{n}| \leq C \varepsilon^{\frac{1}{2}}\left[\int_{D^{2} \backslash D_{1 / 2}^{2}}|\vec{H}|^{2}\right]^{\frac{1}{2}} .
$$

We note that $\vec{v}:=\chi \vec{H}-\vec{v}_{1}-\vec{v}_{2}$ belongs to $L^{2}\left(D^{2}\right)$, and that it satisfies $\mathcal{L}_{\vec{n}} \vec{v}=0$. In addition, since $\chi$ is compactly supported in $D^{2}$, it follows that $\nabla \vec{v}$ is the sum of a compactly supported distribution in the interior of $D^{2}$ and of a function in $L^{2, \infty}$. The trace of $\vec{v}$ on $\partial D^{2}$ is therefore welldefined, and it is in fact equal to zero.

Assuming now that $\nabla \vec{n}$ lies in the Lorentz space $L^{2,1}\left(D^{2}\right)$ enables us to apply Lemma A.8. In particular, we deduce that $\vec{v}$ is identically 0 . Therefore, we have proved that $\nabla(\chi \vec{H}) \in L^{2, \infty}$. More precisely:

$$
\|\nabla(\chi \vec{H})\|_{L^{2, \infty}\left(D^{2}\right)} \leq C\left[\int_{D^{2} \backslash D_{1 / 2}^{2}}|\vec{H}|^{2}\right]^{\frac{1}{2}} .
$$

On $D_{1 / 2}^{2}$, there holds $\mathcal{L}_{\vec{n}}(\chi \vec{H})=\mathscr{L}_{\vec{n}}(\vec{H})=0$. We find helpful to introduce the following Hodge decomposition on $D_{1 / 2}^{2}$ :

$$
\nabla \vec{H}-3 \pi_{\vec{n}}(\nabla \vec{H})=\nabla C+\nabla^{\perp} D+\vec{r},
$$

where $\vec{r}$ is harmonic, and with the boundary conditions $C=0$ on $\partial D_{1 / 2}^{2}$ and $\partial D / \partial v=0$ on $D_{1 / 2}^{2}$. It is not difficult to see that $C$ and $D$ satisfy the systems

$$
\begin{cases}\Delta C=\operatorname{div}\left(\vec{H} \wedge \nabla^{\perp} \vec{n}\right) & \text { in } D_{1 / 2}^{2} \\ C=0 & \text { on } \partial D_{1 / 2}^{2},\end{cases}
$$

and

$$
\begin{cases}\Delta D=3 \operatorname{div}\left(\pi_{\vec{n}}\left(\nabla^{\perp} \vec{H}\right)\right) & \text { in } D_{1 / 2}^{2} \\ \frac{\partial D}{\partial v}=0 & \text { on } \partial D_{1 / 2}^{2} .\end{cases}
$$

The right-hand sides of (III.15) and of (III.16) are Jacobians of $\vec{H}$ and $\vec{n}$. Since $\nabla \vec{n} \in L^{2}\left(D_{1 / 2}^{2}\right)$ and $\nabla \vec{H} \in L^{2, \infty}\left(D_{1 / 2}^{2}\right)$, the Wente-type estimate (III.3) yields

$$
\|\nabla C\|_{L^{2}\left(D_{1 / 2}^{2}\right)}+\|\nabla D\|_{L^{2}\left(D_{1 / 2}^{2}\right)} \leq C\|\nabla \vec{n}\|_{L^{2}\left(D_{1 / 2}^{2}\right)}\|\nabla \vec{H}\|_{L^{2, \infty}\left(D_{1 / 2}^{2}\right)} .
$$

Moreover, because $\vec{r}$ is harmonic, there holds the estimate

$$
\|\vec{r}\|_{L^{2}\left(D_{1 / 4}\right)} \leq C\|\vec{r}\|_{L^{2, \infty}\left(D_{1 / 2}\right)} .
$$


Let us also note that

$$
\left|\nabla \vec{H}-3 \pi_{\vec{n}}(\nabla \vec{H})\right| \geq|\nabla \vec{H}| .
$$

Compiling now (III.17), (III.18), and (III.19) into (III.14) implies

$$
\|\nabla \vec{H}\|_{L^{2}\left(D_{1 / 4}^{2}\right)} \leq C\|\nabla \vec{n}\|_{L^{2}\left(D_{1 / 2}^{2}\right)}\|\nabla \vec{H}\|_{L^{2, \infty}\left(D_{1 / 2}^{2}\right)}+\|\nabla \vec{H}\|_{L^{2, \infty}\left(D_{1 / 2}^{2}\right)} .
$$

We may consider a Hodge decomposition analogous to (III.14), but on $D_{1 / 4}^{2}$ in place of $D_{1 / 2}^{2}$. For this new decomposition, the Wente inequality ${ }^{6}$ (3.47) in Theorem 3.4.1 from [Hel] may be used in place of (III.3). Arguing mutatis mutandis as we did above then yields the estimate

$$
\|\nabla \vec{H}\|_{L^{2,1}\left(D_{1 / 8}^{2}\right)} \leq C\|\nabla \vec{n}\|_{L^{2}\left(D_{1 / 4}^{2}\right)}\|\nabla \vec{H}\|_{L^{2}\left(D_{1 / 4}^{2}\right)}+\|\nabla \vec{H}\|_{L^{2, \infty}\left(D_{1 / 4}^{2}\right)} .
$$

As explained in [Hel], $\nabla \lambda$ belongs to $L^{2,1}\left(D^{2}\right)$. From (II.9), it thus follows that $\nabla \vec{n}$ is in $L^{\infty}\left(D_{1 / 8}^{2}\right)$. Moreover, via combining (III.13), (III.20), and (III.21), we infer that

$$
\|\nabla \vec{n}\|_{L^{\infty}\left(D_{1 / 8}^{2}\right)}^{2} \leq C \int_{D^{2}}|\nabla \vec{n}|^{2} .
$$

This last estimate can now easily be bootstrapped within the Willmore equation so as to obtain (I.16) for general $k$. This concludes the proof of Theorem I.5.

\section{Point removability for Willmore graphs}

This section is devoted to proving the statement of Theorem I.6.

As explained in [KS3, pp. 332-334], under the assumptions of Theorem I.6, we may consider the Lipschitz conformal parametrization $\vec{\Phi}$ obtained by following arguments based on Huber's result on conformal parametrizations of complete surfaces in $\mathbb{R}^{m}$ [Hub], along with the estimates given in the work by Müller and Sverák [MS].

If our surface is Willmore outside of some point (to be removed), then the pre-image of that point under the map $\vec{\Phi}$ must lie inside $D^{2}$. Applying, if necessary, an appropriate Möbius transformation, we may assume without loss of generality that the point to be removed has pre-image 0 . According to Theorem I.3, we know that $\vec{\Phi}$ is $C^{\infty}$ in $D^{2} \backslash\{0\}$. Moreover, it follows from (I.16) that there exists a positive function $\delta(r)$ such that $\lim _{r \downarrow 0} \delta(r)=0$ and

$$
|x||\nabla \vec{n}(x)|+|x|^{2}\left|\nabla^{2} \vec{n}(x)\right| \leq \delta(|x|) \quad \forall x \in D^{2} \backslash\{0\} .
$$

6 Originally derived by Luc Tartar in [Ta1]. 
The distances are understood in the flat metric on $D^{2}$. Owing to some estimates on the function $\lambda$ (defined at the beginning of Subsect. II.1) proved in [MS], that distance is equivalent to the distance with respect to the induced metric.

Given $\varepsilon>0$, we may restrict our attention to a smaller disk and apply an appropriate dilation, so as to obtain from the property of $\delta(|x|)$ and from (IV.1) the estimate

$$
\||x||\nabla \vec{n}|(x)\|_{\infty}^{2}+\int_{D^{2}}|\nabla \vec{n}|^{2} \leq \varepsilon .
$$

Next, since $\vec{H}$ belongs to $L^{2}\left(D^{2}\right)$, the distribution $\mathcal{L}_{\vec{n}} \vec{H}$ makes sense in $\mathscr{D}^{\prime}\left(D^{2}\right)$. In addition, because $\vec{\Phi}$ is Willmore in $D^{2} \backslash\{0\}$ by hypothesis, the distribution $\mathscr{L}_{\vec{n}} \vec{H}$ is supported on zero, and it is thus a finite linear combination of derivatives of the Dirac mass at the origin. Yet, as $\mathcal{L}_{\vec{n}} \vec{H}$ is a sum of an $H^{-2}$ distribution and of derivatives of $L^{1}$ functions, it ensues that $\mathcal{L}_{\vec{n}} \vec{H}$ must in fact be proportional to the Dirac mass centered at the origin; namely:

$$
\mathcal{L}_{\vec{n}} \vec{H}=\vec{c}_{0} \delta_{0}
$$

In anticipation of our ultimate goal, we introduce the constant $\vec{H}_{0}$ defined via $\vec{c}_{0}=-4 \pi \vec{H}_{0}$. Per Lemma A.4 from the appendix, let now $\vec{L}$ solve the problem

$$
\begin{cases}\mathcal{L}_{\vec{n}} \vec{L}=-4 \pi \vec{H}_{0} & \text { in } D^{2}, \\ \vec{L}=0 & \text { on } \partial D^{2} .\end{cases}
$$

We know that $\nabla \vec{L} \in L^{2, \infty}$. Since $\vec{n}$ is smooth over $D^{2} \backslash\{0\}$, and since it satisfies $\left\||x|^{k} \nabla^{k} \vec{n}\right\|_{L^{\infty}\left(D^{2}\right)}<+\infty$, we can apply Lemma A.9 from the appendix in each annulus $D_{2^{-i}}^{2} \backslash D_{2^{-i-1}}^{2}$, for $i \in \mathbb{N}$, to deduce that $\vec{L}$ is in fact smooth in $D^{2} \backslash\{0\}$ and that

$$
\sup _{x \in D^{2}}|x||\nabla \vec{L}(x)|<+\infty .
$$

As in the previous section, we find helpful to introduce the cut-off function $\chi$ compactly supported in $D^{2}$ and assuming the value 1 on $D_{1 / 2}^{2}$. We also introduce the functions $\vec{g}_{1}$ and $\vec{g}_{2}$, as we did in (III.7) and (III.8). And we consider again the functions $\vec{v}_{1}$ and $\vec{v}_{2}$ whose properties are inventoried in the paragraph following (III.10). In particular, according to (III.11) and (III.12), we note that $\nabla \vec{v}_{1}$ and $\nabla \vec{v}_{2}$ are in $L^{2, \infty}$. Furthermore, just as we did for $\vec{L}$ to deduce (IV.5), because $\mathcal{L}_{\vec{n}} \vec{v}_{i}=0$ on $D_{1 / 2}^{2}$ and because $\vec{g}_{1}$ and $\vec{g}_{2}$ are smooth, we have for $i \in \mathbb{N}$ that

$$
\sup _{x \in D^{2}}|x|\left|\nabla \vec{v}_{i}(x)\right|<+\infty .
$$


Let $\vec{w}:=\vec{H}-\vec{v}_{1}-\vec{v}_{2}-\vec{L}$. Clearly, $\vec{w}$ belongs to $L^{2}$ and it solves $\mathscr{L}_{\vec{n}} \vec{w}=0$. It further is smooth on $D^{2} \backslash\{0\}$ and is equal to 0 on $\partial D^{2}$. We claim that $\vec{w}$ is in fact identically 0 on $D^{2}$. We shall now verify this fact.

For $r>0$, let $\chi_{r}(x)=\chi(x / r)$. We consider a sequence $\vec{\phi}_{i}$ of normalized eigenvectors of $\mathcal{L}_{\vec{n}}$ in $W_{0}^{1,2}\left(D^{2}, \mathbb{R}^{m}\right)$, with corresponding eigenvalue $\lambda_{i}$, and forming an orthonormal Hilbert basis ${ }^{7}$ of $L^{2}\left(D^{2}, \mathbb{R}^{m}\right)$. Lemma A.1 from the appendix guarantees that $\lambda_{i} \neq 0$. Furthermore, there holds:

$$
\begin{aligned}
\int_{D^{2}}\left(1-\chi_{r}\right) \vec{w} \cdot \vec{\phi}_{i}= & \frac{1}{\lambda_{i}} \int_{D^{2}}\left(1-\chi_{r}\right) \vec{w} \cdot \mathcal{L}_{\vec{n}} \vec{\phi}_{i} \\
= & \frac{1}{\lambda_{i}} \int_{D^{2}} \nabla \chi_{r} \vec{w} \cdot\left[\nabla \vec{\phi}_{i}-3 \pi_{\vec{n}}\left(\nabla \vec{\phi}_{i}\right)-\vec{\phi}_{i} \wedge \nabla^{\perp} \vec{n}\right] \\
& -\frac{1}{\lambda_{i}} \int_{D^{2}}\left(1-\chi_{r}\right) \nabla \vec{w} \cdot\left[\nabla \vec{\phi}_{i}-3 \pi_{\vec{n}}\left(\nabla \vec{\phi}_{i}\right)-\vec{\phi}_{i} \wedge \nabla^{\perp} \vec{n}\right] \\
= & \frac{1}{\lambda_{i}} \int_{D^{2}}\left(1-\chi_{r}\right) \vec{\phi}_{i} \cdot \mathcal{L}_{\vec{n}} \vec{w} \\
& +\frac{1}{\lambda_{i}} \int_{D^{2}} \nabla \chi_{r} \vec{w} \cdot\left[\nabla \vec{\phi}_{i}-3 \pi_{\vec{n}}\left(\nabla \vec{\phi}_{i}\right)\right] \\
& -\frac{1}{\lambda_{i}} \int_{D^{2}} \nabla \chi_{r}\left[\nabla \vec{w}-3 \pi_{\vec{n}}(\nabla \vec{w})-\vec{w} \wedge \nabla^{\perp} \vec{n}\right] \cdot \vec{\phi}_{i} .
\end{aligned}
$$

Since $\mathscr{L}_{\vec{n}} \vec{w}=0$ holds in the distributional sense, we find that

$$
-\int_{D^{2}} \nabla \chi_{r}\left[\nabla \vec{w}-3 \pi_{\vec{n}}(\nabla \vec{w})-\vec{w} \wedge \nabla^{\perp} \vec{n}\right]=\left\langle\mathcal{L}_{\vec{n}} \vec{w}, \chi_{r}\right\rangle_{D^{\prime}, C_{0}^{\infty}}=0 .
$$

Thus, in the last term of (IV.7), we can subtract from $\vec{\phi}_{i}$ the vector $\vec{c}_{r, i}$, which is the average of $\vec{\phi}_{i}$ over $D_{r}^{2} \backslash D_{r / 2}^{2}$, without modifying the equalities. Namely:

$$
\begin{aligned}
& \int_{D^{2}}\left(1-\chi_{r}\right) \vec{w} \cdot \vec{\phi}_{i}= \frac{1}{\lambda_{i}} \int_{D^{2}} \nabla \chi_{r} \vec{w} \cdot\left[\nabla \vec{\phi}_{i}-3 \pi_{\vec{n}}\left(\nabla \vec{\phi}_{i}\right)\right] \\
&-\frac{1}{\lambda_{i}} \int_{D^{2}} \nabla \chi_{r}\left[\nabla \vec{w}-3 \pi_{\vec{n}}(\nabla \vec{w})-\vec{w} \wedge \nabla^{\perp} \vec{n}\right] \\
& \cdot\left(\vec{\phi}_{i}-\vec{c}_{r, i}\right) .
\end{aligned}
$$

Let $v(r):=\sup _{r / 2<|x|<r}|x|^{2}|\nabla \vec{w}|(x)+|x||w|(x)$. This quantity enables us to control the right-hand side of (IV.9), as we shall now demonstrate. Indeed,

7 Whose existence is inferred from Lemma A.1 and the theorem of Hilbert-Schmidt. 
using the Cauchy-Schwarz inequality, we have first that

$$
\begin{aligned}
& \left|\frac{1}{\lambda_{i}} \int_{D^{2}} \nabla \chi_{r} \vec{w} \cdot\left[\nabla \vec{\phi}_{i}-3 \pi_{\vec{n}}\left(\nabla \vec{\phi}_{i}\right)\right]\right| \\
& \quad \leq C_{i} \int_{D_{r}^{2} \backslash D_{r / 2}^{2}} \frac{v(r)}{r^{2}}\left|\nabla \vec{\phi}_{i}\right| \\
& \quad \leq C_{i} v(r)\left[\int_{D_{r}^{2} \backslash D_{r / 2}^{2}} \frac{\left|\nabla \vec{\phi}_{i}\right|^{2}}{|x|^{2}}\right]^{\frac{1}{2}} .
\end{aligned}
$$

Using Lemma A.5 and the fact that $\lim _{r \backslash 0} v(r)=0$, which ensues from combining altogether (IV.1), (IV.5), and (IV.6), we note that the left-hand side of (IV.10) converges to 0 as $r \searrow 0$.

On the other hand, with the help of the Cauchy-Schwarz and of the Poincaré inequalities, we find that

$$
\begin{aligned}
& \left|-\frac{1}{\lambda_{i}} \int_{D^{2}} \nabla \chi_{r}\left[\nabla \vec{w}-3 \pi_{\vec{n}}(\nabla \vec{w})-\vec{w} \wedge \nabla^{\perp} \vec{n}\right] \cdot\left(\vec{\phi}_{i}-\vec{c}_{r, i}\right)\right| \\
& \quad \leq C_{i} \int_{D_{r}^{2} \backslash D_{r / 2}^{2}}\left[\frac{v(r)}{r^{3}}+\frac{v(r) \delta(r)}{r^{3}}\right]\left|\vec{\phi}_{i}-\vec{c}_{r, i}\right| \\
& \quad \leq C_{i}[v(r)+v(r) \delta(r)] \frac{1}{r^{2}}\left[\int_{D_{r}^{2} \backslash D_{r / 2}^{2}}\left|\vec{\phi}_{i}-\vec{c}_{r, i}\right|^{2}\right]^{\frac{1}{2}} \\
& \quad \leq C_{i}[v(r)+v(r) \delta(r)]\left[\int_{D_{r}^{2} \backslash D_{r / 2}^{2}} \frac{\left|\nabla \vec{\phi}_{i}\right|^{2}}{|x|^{2}}\right]^{\frac{1}{2}} .
\end{aligned}
$$

Calling again upon Lemma A.5, and because $v(r)$ and $\delta(r)$ both tend to zero as $r \searrow 0$, it follows likewise that the left-hand side of (IV.11) tends to 0 as $r$ decreases to zero. Altogether, (IV.9), (IV.10), and (IV.11) thus imply that $\int_{D^{2}}\left(1-\chi_{r}\right) \vec{w} \cdot \vec{\phi}_{i}$ converges to zero as $r$ tends to zero. In turn, this yields that $\int_{D^{2}} \vec{w} \cdot \vec{\phi}_{i}=0$. Since this holds for every $i$, and since $\vec{\phi}_{i}$ is an orthonormal basis of $L^{2}$, we conclude at once that $\vec{w}$ is indeed identically zero, and hence:

$$
\vec{H}=\vec{L}+\vec{v}_{1}+\vec{v}_{2} .
$$

We have previously seen that $\mathcal{L}_{\vec{n}} \vec{v}_{i}=0$ on $D_{1 / 2}^{2}$. From Lemma A.9, we whence deduce that $\vec{v}_{1}$ and $\vec{v}_{2}$ are smooth on $D_{1 / 2}^{2}$. It thus appears natural to study next the asymptotic expansion of $\vec{H}$ near the origin. To this end, we first observe that $\nabla \vec{H} \in L^{2, \infty}$, which follows from $\nabla \vec{L} \in L^{2, \infty}$. Then using (II.11), we infer that

$$
\Delta \nabla \vec{\Phi}=4 e^{2 \lambda} \vec{H} \nabla \lambda+2 e^{2 \lambda} \nabla \vec{H} \in \bigcap_{p<2} L^{p} .
$$


Since $e^{\lambda}=|\nabla \vec{\Phi}|$, we conclude from the latter that $\nabla e^{\lambda} \in L^{q}$ for every $q<+\infty$. Bootstrapping this fact back into (IV.13) then implies that $\Delta \nabla \vec{\phi} \in L^{2, \infty}$, thereby showing that $\nabla^{2} \vec{n} \in L^{2, \infty}$. Inspecting the proof of Theorem 5.1.1 from [Hel], we find that the Coulomb framing $\left\{\vec{e}_{1}, \vec{e}_{2}\right\}$ bears the same regularity as that of $\vec{n}$. Whence $\nabla^{2} \vec{e}_{i} \in L^{2, \infty}$. This yields in particular that $\vec{e}_{i} \in C^{0, \alpha}$ for every $0<\alpha<1$ and $i=1,2$.

We claim now that $e_{i}(0) \cdot \vec{H}_{0}=0$. To see why this must be true, note firstly that $\vec{H} \cdot \vec{e} \equiv 0$ implies:

$$
\begin{aligned}
-4 \pi \vec{e}_{i}(0) \cdot \vec{H}_{0} \delta_{0}= & \vec{e}_{i} \cdot \operatorname{div}\left(\nabla \vec{H}-3 \pi_{\vec{n}}(\nabla \vec{H})-\vec{H} \wedge \nabla^{\perp} \vec{n}\right) \\
= & \operatorname{div}\left(\vec{e}_{i} \cdot \nabla \vec{H}-3 \vec{e}_{i} \cdot \vec{H} \wedge \nabla^{\perp} \vec{n}\right) \\
& -\nabla \vec{e}_{i} \cdot\left[\nabla \vec{H}-3 \pi_{\vec{n}}(\nabla \vec{H})-\vec{H} \wedge \nabla^{\perp} \vec{n}\right] \\
= & \operatorname{div}\left(-\vec{H} \cdot \nabla \vec{e}_{i}-3 \vec{e}_{i} \cdot \vec{H} \wedge \nabla^{\perp} \vec{n}\right) \\
& -\nabla \vec{e}_{i} \cdot\left[\nabla \vec{H}-3 \pi_{\vec{n}}(\nabla \vec{H})-\vec{H} \wedge \nabla^{\perp} \vec{n}\right] .
\end{aligned}
$$

Observe next that the right-hand side of (IV.14) belongs to $L^{p}$, for some $p>1$, and that it should be proportional to the Dirac mass at the origin. Hence the coefficient $4 \pi \vec{e}_{i}(0) \cdot \vec{H}_{0}$ must be zero, which settles at once the desired claim.

Letting $\vec{R}:=\vec{L}-\vec{H}_{0} \log |x|$, there holds:

$$
\mathcal{L}_{\vec{n}} \vec{R}=-3 \operatorname{div}\left(\pi_{\vec{n}}\left(\vec{H}_{0}\right) \nabla \log |x|\right)-\nabla \log |x| \vec{H}_{0} \wedge \nabla^{\perp} \vec{n} .
$$

Since $\pi_{\vec{n}}\left(\vec{H}_{0}\right)=\left(\vec{H}_{0} \cdot \vec{e}_{1}\right) \vec{e}_{1}+\left(\vec{H}_{0} \cdot \vec{e}_{2}\right) \vec{e}_{2}=\left(\vec{H}_{0} \cdot\left(\vec{e}_{1}-\vec{e}_{1}(0)\right)\right) \vec{e}_{1}+$ $\left(\vec{H}_{0} \cdot\left(\vec{e}_{2}-\vec{e}_{2}(0)\right)\right) \vec{e}_{2}$, and since $\vec{e}_{i} \in C^{0, \alpha}$ for every $\alpha<1$, as explained above, it thus follows that $r^{-1} \pi_{\vec{n}}\left(\vec{H}_{0}\right) \in L^{p}$ for every $p<+\infty$. We have therefore proved that $\mathcal{L}_{\vec{n}} \vec{R} \in W^{-1, p}$ for every $p<+\infty$. Arguing as is done in the proof of Lemma A.1, we find that $\vec{R} \in \bigcap_{p<+\infty} W^{1, p}$, so that $\vec{H}-\vec{H}_{0} \log |x|$ is in $C^{0, \alpha}$ for every $\alpha<1$. This concludes at once the proof of Theorem I.5.

\section{A Appendix}

Lemma A.1 There exists $\varepsilon_{0}>0$ such that for every $0<\varepsilon<\varepsilon_{0}$, a positive constant $C$ independent of $\varepsilon$ exists, with the following property. For $\vec{n}$ from $D^{2}$ into the space of unit $(m-2)$-vectors in $\mathbb{R}^{m}$, suppose there holds the bound:

$$
\int_{D^{2}}|\nabla \vec{n}|^{2} d x \leq \varepsilon .
$$


Let $\vec{g}$ be an arbitrary distribution in the Sobolev space $H^{-1}\left(D^{2}, \mathbb{R}^{m}\right)$, dual to $W_{0}^{1,2}\left(D^{2}, \mathbb{R}^{m}\right)$. Then there exists a unique map $\vec{v}$ in $W_{0}^{1,2}\left(D^{2}, \mathbb{R}^{m}\right)$ such that

$$
\begin{cases}\Delta \vec{v}-3 \operatorname{div}\left(\pi_{\vec{n}}(\nabla \vec{v})\right)-\operatorname{div}\left(\vec{v} \wedge \nabla^{\perp} \vec{n}\right)=\vec{g} & \text { in } D^{2} \\ \vec{v}=0 & \text { on } \partial D^{2}\end{cases}
$$

and

$$
\int_{D^{2}}|\nabla \vec{v}|^{2} \leq C\|\vec{g}\|_{H^{-1}}^{2}
$$

Moreover, the operator $\mathcal{L}_{\vec{n}}^{-1}: \vec{g} \mapsto \vec{v}$ is self-adjoint and compact from $L^{2}\left(D^{2}, \mathbb{R}^{m}\right)$ into itself.

Prior to proving Lemma A.1, it is helpful to establish first the following intermediary result.

Lemma A.2 There exists $\varepsilon_{0}>0$ such that for every $0<\varepsilon<\varepsilon_{0}$, a positive constant $C$ independent of $\varepsilon$ exists, with the following property. For $\vec{n}$ from $D^{2}$ into the space of unit $(m-2)$-vectors in $\mathbb{R}^{m}$, suppose there holds the bound:

$$
\int_{D^{2}}|\nabla \vec{n}|^{2} d x \leq \varepsilon
$$

Let $\vec{g}$ be an arbitrary distribution in the Sobolev space $H^{-1}\left(D^{2}, \mathbb{R}^{m}\right)$, dual to $W_{0}^{1,2}\left(D^{2}, \mathbb{R}^{m}\right)$. Then there exists a unique map $\vec{v}$ in $W_{0}^{1,2}\left(D^{2}, \mathbb{R}^{m}\right)$ such that

$$
\begin{cases}\Delta \vec{v}-3 \operatorname{div}\left(\pi_{\vec{n}}(\nabla \vec{v})\right)=\vec{g} & \text { in } D^{2} \\ \vec{v}=0 & \text { on } \partial D^{2}\end{cases}
$$

and

$$
\int_{D^{2}}|\nabla \vec{v}|^{2} \leq C\|\vec{g}\|_{H^{-1}}^{2}
$$

Proof of Lemma A.2. We first show that under the hypothesis (A.4), the following implication holds for every $\vec{C}$ in $W^{1,2}\left(D^{2}, \mathbb{R}^{m}\right)$ :

$$
\left\{\begin{array}{ll}
\Delta \vec{C}-3 \operatorname{div}\left(\pi_{\vec{n}}(\nabla \vec{C})\right)=0 & \text { in } D^{2} \\
\vec{C}=0 & \text { on } \partial D^{2}
\end{array} \Longrightarrow \vec{C} \equiv 0 .\right.
$$

Since

$$
\operatorname{div}\left(\nabla \vec{C}-3 \pi_{\vec{n}}(\nabla \vec{C})\right)=0
$$


the Poincaré lemma guarantees that there exists $\vec{D}$ in $W^{1,2}\left(D^{2}, \mathbb{R}^{m}\right)$ satisfying

$$
\nabla^{\perp} \vec{D}=\nabla \vec{C}-3 \pi_{\vec{n}}(\nabla \vec{C})
$$

This implies in particular that $\vec{D}$ is a $W^{1,2}$ solution of the problem

$$
\begin{cases}\Delta \vec{D}=3 \sum_{k=1}^{m} \nabla^{\perp} C^{k} \cdot \nabla\left(e_{1}^{k} \vec{e}_{1}\right)+3 \sum_{k=1}^{m} \nabla^{\perp} C^{k} \cdot \nabla\left(e_{2}^{k} \vec{e}_{2}\right) & \text { in } D^{2} \\ \frac{\partial \vec{D}}{\partial \nu}=0 & \text { on } \partial D^{2}\end{cases}
$$

where $C^{k}$ are the coordinates of $\vec{C}$ in the canonical basis of $\mathbb{R}^{m}$. Here $\left\{\vec{e}_{1}, \vec{e}_{2}\right\}$ is an orthonormal basis of the 2-dimensional subspace defined by its normal $\vec{n}$. It is explicitly given in Lemma 5.1.4 from [Hel]. That same lemma also states that there exists some constant $C$ such that

$$
\int_{D^{2}}\left|\nabla \vec{e}_{1}\right|^{2}+\left|\nabla \vec{e}_{2}\right|^{2} d x \leq C \int_{D^{2}}|\nabla \vec{n}|^{2} d x .
$$

A Wente-type $W^{1,2}$ estimate derived in Lemma 3.1.2 from [Hel] may be adapted $^{8}$ to our case so as to obtain the estimate

$$
\begin{aligned}
\int_{D^{2}}|\nabla \vec{D}|^{2} d x & \leq C_{1}\left[\int_{D^{2}}\left|\nabla \vec{e}_{1}\right|^{2}+\left|\nabla \vec{e}_{2}\right|^{2} d x\right] \int_{D^{2}}|\nabla \vec{C}|^{2} d x \\
& \leq C_{1} \varepsilon \int_{D^{2}}|\nabla \vec{C}|^{2} d x
\end{aligned}
$$

On the other hand, let us note that (A.8) implies:

$$
|\nabla \vec{D}|^{2}=\left|\pi_{T}(\nabla \vec{C})\right|^{2}+4\left|\pi_{\vec{n}}(\nabla \vec{C})\right|^{2} \geq|\nabla \vec{C}|^{2},
$$

where $\pi_{T}$ denotes the orthogonal projection on the 2-plane in $\mathbb{R}^{m}$ defined by the normal $\vec{n}$. Upon combining (A.11) and (A.12), we then deduce, for $\varepsilon<1 /\left(2 C_{1}\right)$, that $\vec{C} \equiv 0$, thereby establishing the implication (A.7).

Let now $\vec{g} \in H^{-1}\left(D^{2}, \mathbb{R}^{m}\right)$ and $\vec{B}$ satisfy

$$
\begin{cases}\Delta \vec{B}=\vec{g} & \text { in } D^{2} \\ \vec{B}=0 & \text { on } \partial D^{2}\end{cases}
$$

${ }^{8}$ Hélein considers a problem with Dirichlet boundary condition, whereas we are concerned with Neumann boundary condition. However, a classical argument from elliptic theory allows to pass from the former setting to the latter, and derive analogous estimates. 
We claim that there exists $(\vec{A}, \vec{F})$ such that

$$
\begin{cases}\operatorname{div} \vec{F}=\operatorname{div}\left(\pi_{T}\left(\nabla^{\perp} \vec{A}\right)-\frac{1}{2} \pi_{\vec{n}}\left(\nabla^{\perp} A\right)\right) & \text { in } D^{2} \\ \operatorname{curl} \vec{F}=-\operatorname{curl}\left(\pi_{T}(\nabla \vec{B})-\frac{1}{2} \pi_{\vec{n}}(\nabla \vec{B})\right) & \text { in } D^{2} \\ \vec{F} \cdot v=0 & \text { on } \partial D^{2}\end{cases}
$$

where $\vec{A}$ is the curl-part in the Hodge decomposition of $\pi_{T}(\vec{F})-2 \pi_{\vec{n}}(\vec{F})$ given by:

$$
\begin{cases}-\Delta \vec{A}=\operatorname{curl}\left(\pi_{T}(\vec{F})-2 \pi_{\vec{n}}(\vec{F})\right) & \text { in } D^{2} \\ \vec{A}=0 & \text { on } \partial D^{2}\end{cases}
$$

We attract the reader's attention on the fact that in (A.14), $\vec{F} \cdot v$ is an element of $\mathbb{R}^{m}$. This is because $\vec{F} \in \mathbb{R}^{2} \otimes \mathbb{R}^{m}$ and $v \in \mathbb{R}^{2} \otimes \mathbb{R}$. The dot product in $\vec{F} \cdot v$ is understood to act as scalar multiplication on the $\mathbb{R}^{2}$ component, and as usual multiplication on the second component.

The existence of a solution $(\vec{A}, \vec{F})$ to the system (A.14)-(A.15) is again a consequence of Wente's estimate. More precisely, we write on one hand:

$$
\begin{aligned}
\operatorname{div}\left(\pi_{T}\left(\nabla^{\perp} \vec{A}\right)-\frac{1}{2} \pi_{\vec{n}}\left(\nabla^{\perp} A\right)\right)= & \frac{3}{2} \sum_{k=1}^{m} \nabla^{\perp} A^{k} \cdot \nabla\left(e_{1}^{k} \vec{e}_{1}\right) \\
& +\frac{3}{2} \sum_{k=1}^{m} \nabla^{\perp} A^{k} \cdot \nabla\left(e_{2}^{k} \vec{e}_{2}\right) ;
\end{aligned}
$$

and on the other hand:

$$
\begin{aligned}
\operatorname{curl}\left(\pi_{T}(\nabla \vec{B})-\frac{1}{2} \pi_{\vec{n}}(\nabla \vec{B})\right)= & \frac{3}{2} \sum_{k=1}^{m} \nabla B^{k} \cdot \nabla^{\perp}\left(e_{1}^{k} \vec{e}_{1}\right) \\
& +\frac{3}{2} \sum_{k=1}^{m} \nabla B^{k} \cdot \nabla^{\perp}\left(e_{2}^{k} \vec{e}_{2}\right),
\end{aligned}
$$

where $A^{k}$ and $B^{k}$ are the coordinates of $\vec{A}$ and $\vec{B}$. Therefore, using Wente's estimate, we obtain the a-priori inequalities

$$
\begin{aligned}
\int_{D^{2}}|\vec{F}|^{2} & \leq C_{2}\left[\int_{D^{2}}\left|\nabla \vec{e}_{1}\right|^{2}+\left|\nabla \vec{e}_{2}\right|^{2} d x\right] \int_{D^{2}}|\nabla \vec{A}|^{2}+|\nabla \vec{B}|^{2} d x \\
& \leq C_{2} \varepsilon \int_{D^{2}}|\nabla \vec{A}|^{2}+|\nabla \vec{B}|^{2} d x .
\end{aligned}
$$


From (A.15) and standard elliptic estimates, we also have that

$$
\int_{D^{2}}|\nabla \vec{A}|^{2} d x \leq C_{3} \int_{D^{2}}|\vec{F}|^{2} d x .
$$

Thus, for $C_{3} C_{2} \varepsilon<1 / 2$, a standard fixed-point argument yields the existence and uniqueness of $(\vec{A}, \vec{F})$ satisfying (A.14) and (A.15).

Since

$$
\operatorname{div}\left(\vec{F}-\left(\pi_{T}\left(\nabla^{\perp} \vec{A}\right)-\frac{1}{2} \pi_{\vec{n}}\left(\nabla^{\perp} \vec{A}\right)\right)\right)=0,
$$

there exists $\vec{C}$ in $W_{0}^{1,2}\left(D^{2}, \mathbb{R}^{m}\right)$ with

$$
\vec{F}-\left(\pi_{T}\left(\nabla^{\perp} \vec{A}\right)-\frac{1}{2} \pi_{\vec{n}}\left(\nabla^{\perp} A\right)\right)=\nabla^{\perp} \vec{C} .
$$

Hence we deduce that

$$
\pi_{T}(\vec{F})-2 \pi_{\vec{n}}(\vec{F})=\nabla^{\perp} \vec{A}+\pi_{T}\left(\nabla^{\perp} \vec{C}\right)-2 \pi_{\vec{n}}\left(\nabla^{\perp} \vec{C}\right) .
$$

Applying the curl operator throughout both sides of the latter, and calling upon (A.15), we infer that $\vec{C}$ solves the problem

$$
\begin{cases}\Delta \vec{C}-3 \operatorname{div}\left(\pi_{\vec{n}}(\nabla \vec{C})\right)=-\operatorname{curl}\left(\pi_{T}\left(\nabla^{\perp} \vec{C}\right)-2 \pi_{\vec{n}}\left(\nabla^{\perp} \vec{C}\right)\right)=0 & \text { in } D^{2} \\ \vec{C}=0 & \text { on } \partial D^{2} .\end{cases}
$$

Then (A.7) implies that $\vec{C} \equiv 0$, and hence that

$$
\pi_{T}(\vec{F})-2 \pi_{\vec{n}}(\vec{F})=\nabla^{\perp} \vec{A} .
$$

From (A.14), there exists $\vec{v}$ in $W_{0}^{1,2}\left(D^{2}, \mathbb{R}^{m}\right)$ satisfying

$$
\vec{F}=-\pi_{T}(\nabla \vec{B})+\frac{1}{2} \pi_{\vec{n}}(\nabla \vec{B})+\nabla \vec{v} .
$$

Upon combining (A.23) and (A.24) together, it then follows that

$$
\nabla^{\perp} \vec{A}=-\nabla \vec{B}+\pi_{T}(\nabla \vec{v})-2 \pi_{\vec{n}}(\nabla \vec{v}) .
$$

Comparing this identity with (A.13), we conclude that $\vec{v}$ solves (A.5). From (A.7), this is the unique solution. Finally, (A.6) follows at once from (A.18) and (A.19). This completes the proof of Lemma A.2.

Proof of Lemma A.1. Let $\Delta_{0}^{-1}$ denote the continuous isomorphism from $H^{-1}\left(D^{2}, \mathbb{R}^{m}\right)$ into $W_{0}^{1,2}\left(D^{2}, \mathbb{R}^{m}\right)$, which to some distribution $\vec{g}$ in 
$H^{-1}\left(D^{2}, \mathbb{R}^{m}\right)$ assigns the solution $\vec{v}$ of

$$
\begin{cases}\Delta \vec{v}=\vec{g} & \text { in } D^{2} \\ \vec{v}=0 & \text { on } \partial D^{2}\end{cases}
$$

We have seen in Lemma A.2 that the operator $\mathcal{A}_{\vec{n}} \vec{v}:=\Delta \vec{v}-3 \operatorname{div}\left(\pi_{\vec{n}}(\nabla \vec{n})\right)$ is a continuous isomorphism from $W_{0}^{1,2}\left(D^{2}, \mathbb{R}^{m}\right)$ into $H^{-1}\left(D^{2}, \mathbb{R}^{m}\right)$, and that the norms of $\mathcal{A}_{\vec{n}}$ and of $\mathcal{A}_{\vec{n}}^{-1}$ are independent of $\vec{n}$ satisfying (A.4) for $\varepsilon<\varepsilon_{0}$, where $\varepsilon_{0}$ is the universal constant given in Lemma A.2. The invertibility of the operator

$$
\Delta_{0}^{-1} \mathcal{A}_{\vec{n}}(\vec{v})-\Delta_{0}^{-1} \operatorname{div}\left(\vec{v} \wedge \nabla^{\perp} \vec{n}\right),
$$

which maps $W_{0}^{1,2}\left(D^{2}, \mathbb{R}^{m}\right)$ into itself, and with norm independent of $\vec{n}$ satisfying (A.4) for $\varepsilon<\varepsilon_{0}$, is clear. It is indeed a direct consequence of the invertibility of $\Delta_{0}^{-1} \mathcal{A}_{\vec{n}}$ mapping $W_{0}^{1,2}\left(D^{2}, \mathbb{R}^{m}\right)$ into itself, and of the fact that Wente-type estimates (cf. Theorem 3.1.2 of [Hel]) guarantee that the operator $\Delta_{0}^{-1} \operatorname{div}\left(\vec{v} \wedge \nabla^{\perp} \vec{n}\right)$ satisfies

$$
\left\|\Delta_{0}^{-1} \operatorname{div}\left(\vec{v} \wedge \nabla^{\perp} \vec{n}\right)\right\|_{W^{1,2}}^{2} \leq C \int_{D^{2}}|\nabla \vec{v}|^{2} d x \int_{D^{2}}|\nabla \vec{n}|^{2} d x \leq C \varepsilon\|\vec{v}\|_{W^{1,2}}^{2},
$$

for every $\vec{v}$ in $W_{0}^{1,2}\left(D^{2}, \mathbb{R}^{m}\right)$.

We have thus proved the first statement of Lemma A.1, and there only remains to establish the compactness and self-adjointness of the operator $\mathcal{L}_{\vec{n}}^{-1}$ mapping $L^{2}$ into itself. Compactness is clear, since $\mathcal{L}_{\vec{n}}^{-1}$ maps $H^{-1}\left(D^{2}, \mathbb{R}^{m}\right)$ into $W_{0}^{1,2}$, which is a compact subspace of $L^{2}$. Let $\vec{g}$ and $\vec{h}$ belong to $L^{2}\left(D^{2}, \mathbb{R}^{m}\right)$ (chosen to be smooth, for the time being). Upon setting $\vec{v}:=\mathcal{L}_{\vec{n}}^{-1}(\vec{g})$ and $\vec{w}:=\mathcal{L}_{\vec{n}}^{-1}(\vec{h})$, we find:

$$
\begin{aligned}
\int_{D^{2}} & \vec{g} \cdot \mathcal{L}_{\vec{n}}^{-1}(\vec{h}) d x \\
= & \int_{D^{2}} \Delta \vec{v} \cdot \vec{w}-3 \operatorname{div}\left(\pi_{\vec{n}}(\nabla \vec{v})\right) \cdot \vec{w}-\operatorname{div}\left(\vec{v} \wedge \nabla^{\perp} \vec{n}\right) \cdot \vec{w} d x \\
= & \int_{D^{2}} \vec{v} \cdot \Delta \vec{w}+3 \pi_{\vec{n}}(\nabla \vec{v}) \cdot \nabla \vec{w}+\left(\vec{v} \wedge \nabla^{\perp} \vec{n}\right) \cdot \nabla \vec{w} d x \\
= & \int_{D^{2}} \vec{v} \cdot \Delta \vec{w}+3 \nabla \vec{v} \cdot \pi_{\vec{n}}(\nabla \vec{w})-\vec{v} \cdot\left(\nabla \vec{w} \wedge \nabla^{\perp} \vec{n}\right) \\
= & \int_{D^{2}} \vec{v} \cdot \Delta \vec{w}-3 \vec{v} \cdot \operatorname{div}\left(\pi_{\vec{n}}(\nabla \vec{w})\right)-\vec{v} \cdot \operatorname{div}\left(\vec{w} \wedge \nabla^{\perp} \vec{n}\right) \\
= & \int_{D^{2}} \vec{v} \cdot \vec{h}=\int_{D^{2}} \mathcal{L}_{\vec{n}}^{-1}(\vec{g}) \cdot \vec{h} .
\end{aligned}
$$


Via a classical density argument, the latter confirms the self-adjointness of $\mathcal{L}_{\vec{n}}^{-1}$, thereby concluding the proof of Lemma A.1.

We now extend the previous two lemmata to data in $L^{1}$. First of all, we prove

Lemma A.3 There exists $\varepsilon_{0}>0$ such that for every $0<\varepsilon<\varepsilon_{0}$, a constant $C>0$ independent of $\varepsilon$ exists, with the following property. Let $\vec{n}$ from $D^{2}$ into the space of unit $(m-2)$-vectors in $\mathbb{R}^{m}$ satisfy

$$
\int_{D^{2}}|\nabla \vec{n}|^{2} d x \leq \varepsilon
$$

When $\vec{g}$ is an arbitrary map in $L^{1}\left(D^{2}, \mathbb{R}^{m}\right)$, there exists a unique map $\vec{v}$ with $\nabla \vec{v}$ in $L^{2, \infty}\left(D^{2}, \mathbb{R}^{2} \otimes \mathbb{R}^{m}\right)$, and satisfying:

$$
\begin{cases}\Delta \vec{v}-3 \operatorname{div}\left(\pi_{\vec{n}}(\nabla \vec{v})\right)-\operatorname{div}\left(\vec{v} \wedge \nabla^{\perp} \vec{n}\right)=\vec{g} & \text { in } D^{2} \\ \vec{v}=0 & \text { on } \partial D^{2} .\end{cases}
$$

Furthermore, there holds the estimate

$$
\|\nabla \vec{v}\|_{L^{2, \infty}\left(D^{2}\right)} \leq C\|\vec{g}\|_{L^{1}\left(D^{2}\right)} .
$$

Prior to proving Lemma A.3, we shall first establish the following result.

Lemma A.4 There exists $\varepsilon_{0}>0$ such that for every $0<\varepsilon<\varepsilon_{0}$, a constant $C>0$ independent of $\varepsilon$ exists, with the following property. Let $\vec{n}$ from $D^{2}$ into the space of unit $(m-2)$-vectors in $\mathbb{R}^{m}$ satisfy

$$
\int_{D^{2}}|\nabla \vec{n}|^{2} d x \leq \varepsilon
$$

When $\vec{g}$ is an arbitrary map in $L^{1}\left(D^{2}, \mathbb{R}^{m}\right)$, there exists a unique map $\vec{v}$ with $\nabla \vec{v}$ in $L^{2, \infty}\left(D^{2}, \mathbb{R}^{2} \otimes \mathbb{R}^{m}\right)$, and satisfying:

$$
\begin{cases}\Delta \vec{v}-3 \operatorname{div}\left(\pi_{\vec{n}}(\nabla \vec{v})\right)=\vec{g} & \text { in } D^{2} \\ \vec{v}=0 & \text { on } \partial D^{2},\end{cases}
$$

and

$$
\|\nabla \vec{v}\|_{L^{2, \infty}\left(D^{2}\right)} \leq C\|\vec{g}\|_{L^{1}\left(D^{2}\right)} .
$$

Proof of Lemma A.4. It suffices to adapt mutatis mutandis the arguments given in the proof of Lemma A.2. One replaces the estimate (A.11) by its analogous Lorentz-space version given in (III.3); and substitutes the $L^{2, \infty}$ norm in place of the $L^{2}$ norm of the various quantities involved. 
Proof of Lemma A.3. Let $\vec{g}$ be in $L^{1}\left(D^{2}, \mathbb{R}^{m}\right)$. We again set $\mathcal{A}_{\vec{n}} \vec{v}:=$ $\Delta \vec{v}-3 \operatorname{div}\left(\pi_{\vec{n}}(\nabla \vec{v})\right)$. Using Lemma A.4, we first derive the existence of $\vec{v}_{0}$, with $\nabla \vec{v}_{0} \in L^{2, \infty}$, and satisfying

$$
\begin{cases}\mathcal{A}_{\vec{n}} \vec{v}_{0}=\vec{g} & \text { in } D^{2} \\ \vec{v}_{0}=0 & \text { on } \partial D^{2} .\end{cases}
$$

We argue next by induction. Let $\vec{v}_{0}$ be given by (A.35), and $\vec{v}_{k}$, for $k \geq 1$, is the element of $W_{0}^{1,2}\left(D^{2}, \mathbb{R}^{m}\right)$ which solves

$$
\Delta_{0}^{-1} \mathcal{A}_{\vec{n}}\left(\vec{v}_{k}\right)=\Delta_{0}^{-1} \operatorname{div}\left(\vec{v}_{k-1} \wedge \nabla^{\perp} \vec{n}\right),
$$

where $\Delta_{0}^{-1}$ is the operator introduced in (A.26). We know that this problem does have a solution. Indeed, if $\vec{v}_{k-1}$, with $\nabla \vec{v}_{k-1}$ in $L^{2, \infty}$, is given, according to (III.3), we find

$$
\left\|\Delta_{0}^{-1} \operatorname{div}\left(\vec{v}_{k-1} \wedge \nabla^{\perp} \vec{n}\right)\right\|_{W^{1,2}} \leq C\left\|\nabla \vec{v}_{k-1}\right\|_{L^{2, \infty}}\|\nabla \vec{n}\|_{L^{2}} .
$$

Moreover, we have seen in the proof of Lemma A.1 that $\Delta_{0}^{-1} \mathcal{A}_{\vec{n}}$ is a continuous isomorphism of $W_{0}^{1,2}$. Paired to (A.37), this implies the existence and uniqueness of $\vec{v}_{k}$. In addition, there holds:

$$
\left\|\nabla \vec{v}_{k}\right\|_{L^{2, \infty}} \leq\left\|\nabla \vec{v}_{k}\right\|_{L^{2}} \leq C\left\|\nabla \vec{v}_{k-1}\right\|_{L^{2, \infty}}\|\nabla \vec{n}\|_{L^{2}} .
$$

Thus, under the hypothesis that $C\|\nabla \vec{n}\|_{L^{2}}<1 / 2$, the series $\sum_{k=0}^{n} \vec{v}_{k}$ converges to some limit $\vec{v}=\sum_{k=0}^{\infty} \vec{v}_{k}$ solving (A.30) and (A.31). The uniqueness of $\vec{v}$ follows from that of the solution to $\mathcal{A}_{\vec{n}}(\vec{v})=\vec{g}$ for arbitrary $\vec{g} \in L^{1}$ (established in Lemma A.4), and from the aforementioned argument about $C\|\nabla \vec{n}\|_{L^{2}}$ being bounded above by $1 / 2$.

Lemma A.5 There exists $\varepsilon_{0}>0$ such that for every $0<\varepsilon<\varepsilon_{0}$, a constant $C>0$ independent of $\varepsilon$ exists, with the following property. Let $\vec{n}$ from $D^{2}$ into the space of unit $(m-2)$-vectors in $\mathbb{R}^{m}$ satisfy

$$
\||x||\nabla \vec{n}|(x)\|_{L^{\infty}\left(D^{2}\right)}+\int_{D^{2}}|\nabla \vec{n}|^{2} d x \leq \varepsilon .
$$

For some arbitrary $\vec{g} \in L^{2}\left(D^{2}, \mathbb{R}^{m}\right)$, let $\vec{v}$ be the unique map in $W_{0}^{1,2}\left(D^{2}, \mathbb{R}^{m}\right)$ (given by Lemma A.2) such that

$$
\begin{cases}\Delta \vec{v}-3 \operatorname{div}\left(\pi_{\vec{n}}(\nabla \vec{v})\right)-\operatorname{div}\left(\vec{v} \wedge \nabla^{\perp} \vec{n}\right)=\vec{g} & \text { in } D^{2} \\ \vec{v}=0 & \text { on } \partial D^{2} .\end{cases}
$$

We denote by $\vec{v}_{0}$ and $\vec{v}_{\perp}$ the maps from $D^{2}$ into $\mathbb{R}^{m}$ which satisfy

$$
\vec{v}_{0}(x)=\frac{1}{2 \pi|x|} \int_{\partial B_{|x|}(0)} \vec{v} \text { and } \vec{v}_{\perp}=\vec{v}-\vec{v}_{0}
$$


Then there holds:

$$
\left\|\nabla \vec{v}_{0}\right\|_{L^{\infty}\left(D^{2}\right)}^{2}+\int_{D^{2}} \frac{\left|\nabla \vec{v}_{\perp}\right|^{2}}{|x|^{2}}+\int_{D^{2}}\left|\nabla^{2} \vec{v}_{\perp}\right|^{2} \leq C \int_{D^{2}}|\vec{g}|^{2} .
$$

Before proving Lemma A.5, we first establish an intermediate result.

Lemma A.6 Let $a$ and $b$ be two functions, respectively in $W^{2,2}\left(D^{2}, \mathbb{R}\right)$ and in $W^{1,2}\left(D^{2}, \mathbb{R}\right)$, such that $b \in C^{1}\left(D^{2} \backslash\{0\}\right)$ and

$$
\sup _{x \in D^{2} \backslash\{0\}}|x||\nabla b|(x)<+\infty .
$$

Let $\varphi$ be the solution in $W^{1,2}$ of the problem

$$
\begin{cases}\Delta \varphi=\frac{\partial a}{\partial x_{1}} \frac{\partial b}{\partial x_{2}}-\frac{\partial a}{\partial x_{2}} \frac{\partial b}{\partial x_{1}} & \text { in } D^{2} \\ \varphi=0 & \text { on } \partial D^{2}\end{cases}
$$

We denote by $\varphi_{0}$ and $\varphi_{\perp}$ the functions on $D^{2}$ which satsify

$$
\varphi_{0}(x)=\frac{1}{2 \pi|x|} \int_{\partial B_{|x|}(0)} \varphi \text { and } \varphi_{\perp}=\varphi-\varphi_{0} .
$$

Then there holds the inequality:

$$
\begin{aligned}
& \left\|\nabla \varphi_{0}\right\|_{L^{\infty}\left(D^{2}\right)}^{2}+\int_{D^{2}} \frac{\left|\nabla \varphi_{\perp}\right|^{2}}{|x|^{2}}+\int_{D^{2}}\left|\nabla^{2} \varphi_{\perp}\right|^{2} \\
& \quad \leq C\left[\||x||\nabla b|(x)\|_{\infty}^{2}+\int_{D^{2}}|\nabla b|^{2}\right]\left[\int_{D^{2}} \frac{\left|\nabla a_{\perp}\right|^{2}}{|x|^{2}}+\left\|\nabla a_{0}\right\|_{\infty}^{2}\right] .
\end{aligned}
$$

Proof of Lemma A.6. Since $\varphi_{0}$ is the first term in the Fourier decomposition of $\varphi$ (relative to the angular variable), we have

$$
\Delta \varphi_{0}=\frac{\partial a_{0}}{\partial x_{1}} \frac{\partial b_{0}}{\partial x_{2}}-\frac{\partial a_{0}}{\partial x_{2}} \frac{\partial b_{0}}{\partial x_{1}}+\left(\frac{\partial a_{\perp}}{\partial x_{1}} \frac{\partial b_{\perp}}{\partial x_{2}}-\frac{\partial a_{\perp}}{\partial x_{2}} \frac{\partial b_{\perp}}{\partial x_{1}}\right)_{0} .
$$

Indeed, it is clear from the fact that $a_{0}$ and $b_{0}$ depend solely on $|x|$ that

$$
\frac{\partial a_{\perp}}{\partial x_{1}} \frac{\partial b_{0}}{\partial x_{2}}-\frac{\partial a_{\perp}}{\partial x_{2}} \frac{\partial b_{0}}{\partial x_{1}} \text { and } \frac{\partial a_{0}}{\partial x_{1}} \frac{\partial b_{\perp}}{\partial x_{2}}-\frac{\partial a_{0}}{\partial x_{2}} \frac{\partial b_{\perp}}{\partial x_{1}}
$$

have null zeroth-order Fourier coefficient. This follows from the identities

$$
\begin{gathered}
\frac{\partial a_{\perp}}{\partial x_{1}} \frac{\partial b_{0}}{\partial x_{2}}-\frac{\partial a_{\perp}}{\partial x_{2}} \frac{\partial b_{0}}{\partial x_{1}}=\frac{1}{r} \frac{\partial a_{\perp}}{\partial \theta} \dot{b}_{0}(r) \\
\frac{\partial a_{0}}{\partial x_{1}} \frac{\partial b_{\perp}}{\partial x_{2}}-\frac{\partial a_{0}}{\partial x_{2}} \frac{\partial b_{\perp}}{\partial x_{1}}=\dot{a}_{0}(r) \frac{1}{r} \frac{\partial b_{\perp}}{\partial \theta} .
\end{gathered}
$$


For the same reason, the first Jacobian on the right-hand side of (A.46) vanishes. Calling again upon (A.47), we thus find:

$$
\begin{aligned}
\ddot{\varphi}_{0}+\frac{\dot{\varphi}}{r} & =\Delta \varphi_{0}=\left(\frac{\partial a_{\perp}}{\partial x_{1}} \frac{\partial b_{\perp}}{\partial x_{2}}-\frac{\partial a_{\perp}}{\partial x_{2}} \frac{\partial b_{\perp}}{\partial x_{1}}\right)_{0} \\
& =\left(\frac{\partial a_{\perp}}{\partial x_{1}} \frac{\partial b}{\partial x_{2}}-\frac{\partial a_{\perp}}{\partial x_{2}} \frac{\partial b}{\partial x_{1}}\right)_{0} .
\end{aligned}
$$

For notational convenience, let $h(r)$ denote the right-hand side of (A.48). Then we may write $\dot{\varphi}_{0}=r^{-1} \int_{0}^{r} h(s) s d s$. In particular, $\left|\dot{\varphi}_{0}\right|$ may thus be bounded as follows:

$$
\begin{aligned}
\left|\dot{\varphi}_{0}\right| & \leq \frac{1}{2 \pi r} \int_{B_{r}}\left|\nabla a_{\perp}\right||\nabla b| \\
& \leq C\||| x|| \nabla b \mid(x)\|_{\infty}\left[\int_{B_{r}} \frac{\left|\nabla a_{\perp}\right|^{2}}{|x|^{2}}\right]^{\frac{1}{2}} .
\end{aligned}
$$

As for $\varphi_{\perp}$, it satisfies the identity

$$
\begin{aligned}
\Delta \varphi_{\perp} & =\left(\frac{\partial a}{\partial x_{1}} \frac{\partial b}{\partial x_{2}}-\frac{\partial a}{\partial x_{2}} \frac{\partial b}{\partial x_{1}}\right)_{\perp} \\
& =\left(\frac{\partial a_{0}}{\partial x_{1}} \frac{\partial b}{\partial x_{2}}-\frac{\partial a_{0}}{\partial x_{2}} \frac{\partial b}{\partial x_{1}}\right)_{\perp}+\left(\frac{\partial a_{\perp}}{\partial x_{1}} \frac{\partial b}{\partial x_{2}}-\frac{\partial a_{\perp}}{\partial x_{2}} \frac{\partial b}{\partial x_{1}}\right)_{\perp}
\end{aligned}
$$

On one hand, we have

$$
\begin{aligned}
& \int_{D^{2}}\left|\left(\frac{\partial a_{0}}{\partial x_{1}} \frac{\partial b}{\partial x_{2}}-\frac{\partial a_{0}}{\partial x_{2}} \frac{\partial b}{\partial x_{1}}\right)_{\perp}\right|^{2} \\
& \leq \int_{D^{2}}\left|\frac{\partial a_{0}}{\partial x_{1}} \frac{\partial b}{\partial x_{2}}-\frac{\partial a_{0}}{\partial x_{2}} \frac{\partial b}{\partial x_{1}}\right|^{2} \leq C\left\|\nabla a_{0}\right\|_{\infty}^{2} \int_{D^{2}}|\nabla b|^{2} .
\end{aligned}
$$

And on the other hand, there holds

$$
\begin{aligned}
& \int_{D^{2}}\left|\left(\frac{\partial a_{\perp}}{\partial x_{1}} \frac{\partial b}{\partial x_{2}}-\frac{\partial a_{\perp}}{\partial x_{2}} \frac{\partial b}{\partial x_{1}}\right)_{\perp}\right|^{2} \\
& \quad \leq \int_{D^{2}}\left|\frac{\partial a_{\perp}}{\partial x_{1}} \frac{\partial b}{\partial x_{2}}-\frac{\partial a_{\perp}}{\partial x_{2}} \frac{\partial b}{\partial x_{1}}\right|^{2} \leq C\||x||\nabla b|(x)\|_{\infty}^{2} \int_{D^{2}} \frac{\left|\nabla a_{\perp}\right|^{2}}{|x|^{2}} .
\end{aligned}
$$

The desired inequality (A.45) ensues upon combining altogether (A.49), (A.51), and (A.52). This concludes the proof of Lemma A.6. 
Proof of Lemma A.5. Let $\vec{A}, \vec{B}$, and $\vec{C}$ be the solutions of the problems

$$
\begin{gathered}
\begin{cases}\Delta \vec{A}=\vec{g} & \text { in } D^{2} \\
\vec{A}=0 & \text { on } \partial D^{2} ;\end{cases} \\
\begin{cases}\Delta \vec{B}=\operatorname{div}\left(\vec{v} \wedge \nabla^{\perp} \vec{n}\right) & \text { in } D^{2} \\
\vec{B}=0 & \text { on } \partial D^{2} ;\end{cases}
\end{gathered}
$$

and

$$
\begin{cases}\Delta \vec{C}=3 \operatorname{div}\left(\pi_{\vec{n}}\left(\nabla^{\perp} \vec{v}\right)\right) & \text { in } D^{2} \\ \frac{\partial \vec{C}}{\partial v}=0 & \text { on } \partial D^{2}\end{cases}
$$

It is clear that $\vec{v}=\vec{A}+\vec{B}+\vec{C}$. Applying standard elliptic estimates to (A.53), the result of Lemma A.6 to (A.54), and the Neumann boundary condition version of Lemma A.6 to (A.55), we obtain successively the estimates

$$
\begin{gathered}
\left\|\nabla \vec{A}_{0}\right\|_{\infty}^{2}+\int_{D^{2}} \frac{\left|\nabla \vec{A}_{\perp}\right|^{2}}{|x|^{2}}+\int_{D^{2}}\left|\nabla^{2} \vec{A}\right|^{2} \leq C \int_{D^{2}}|\vec{g}|^{2} ; \\
\left\|\nabla \vec{B}_{0}\right\|_{\infty}^{2}+\int_{D^{2}} \frac{\left|\nabla \vec{B}_{\perp}\right|^{2}}{|x|^{2}}+\int_{D^{2}}\left|\nabla^{2} \vec{B}_{\perp}\right|^{2} \leq C \varepsilon\left[\int_{D^{2}} \frac{\left|\nabla \vec{v}_{\perp}\right|^{2}}{|x|^{2}}+\left\|\nabla \vec{v}_{0}\right\|_{\infty}^{2}\right]
\end{gathered}
$$

and

$$
\left\|\nabla \vec{C}_{0}\right\|_{\infty}^{2}+\int_{D^{2}} \frac{\left|\nabla \vec{C}_{\perp}\right|^{2}}{|x|^{2}}+\int_{D^{2}}\left|\nabla^{2} \vec{C}_{\perp}\right|^{2} \leq C \varepsilon\left[\int_{D^{2}} \frac{\left|\nabla \vec{v}_{\perp}\right|^{2}}{|x|^{2}}+\left\|\nabla \vec{v}_{0}\right\|_{\infty}^{2}\right] .
$$

Note that, as in (A.9), we have used the fact that $\operatorname{div}\left(\pi_{\vec{n}}\left(\nabla^{\perp} \vec{v}\right)\right)$ is a Jacobian of the form $-\sum_{k, i} \nabla^{\perp} \vec{v}^{k} \cdot \nabla\left(e_{i}^{k} \vec{e}_{i}\right)$, and that $\left\{\vec{e}_{1}, \vec{e}_{2}\right\}$ is an orthonormal frame generating the 2-plane normal to $\vec{n}$.

Upon choosing $\varepsilon$ small enough, and bringing together (A.56), (A.57), and (A.58), we easily obtain the desired inequality (A.45), thereby concluding the proof of Lemma A.5 is proved.

Lemma A.7 There exists $\varepsilon_{0}>0$ such that for every $0<\varepsilon<\varepsilon_{0}$ the following holds. Let $\vec{n}$ be a $W^{1,2}$ map from $D^{2}$ into the space of unit $(m-2)$ vectors in $\mathbb{R}^{m}$ such that

$$
\int_{D^{2}}|\nabla \vec{n}|^{2} d x \leq \varepsilon
$$


Let $\vec{\phi}$ be a $W^{1,2}$ eigenvector of $\mathcal{L}_{\vec{n}}$, namely, for some constant $\lambda \in \mathbb{R}$ :

$$
\begin{cases}\Delta \vec{\phi}-3 \operatorname{div}\left(\pi_{\vec{n}}(\nabla \vec{\phi})\right)-\operatorname{div}\left(\vec{\phi} \wedge \nabla^{\perp} \vec{n}\right)=\lambda \vec{\phi} & \text { in } D^{2} \\ \vec{\phi}=0 & \text { on } \partial D^{2},\end{cases}
$$

Assume further that the gradient of $\vec{n}$ lies in the Lorentz space $L^{2,1}$.

Then $\vec{\phi}$ is Lipschitz with second derivatives in $L^{2,1}$.

Proof of Lemma A.7. We first prove that $\vec{\phi}$ belongs to $W^{1, p}\left(D^{2}\right)$ for every $1 \leq p<+\infty$. For this, let $2<p<+\infty$, and let $q$ be the constant in $(1,2)$ given by $1 / p=1 / q-1 / 2$, so that $W_{0}^{1, q}\left(D^{2}\right)$ embeds in $L^{p}$. Defining $\vec{g}:=\lambda \vec{\phi} \in L^{q}\left(D^{2}\right)$, we can follow step by step the proof of Lemma A.2, starting from (A.13) and replacing the hypothesis $\vec{g} \in H^{-1}$ by the hypothesis $\vec{g} \in L^{q}$. Doing so, we first observe, with the help of classical elliptic estimates, that

$$
\|\nabla \vec{B}\|_{L^{p}\left(D^{2}\right)} \leq C\|\vec{g}\|_{L^{q}\left(D^{2}\right)} .
$$

Replacing Wente's inequalities by classical $L^{q}$ bound for CalderónZygmund operators, we then obtain the a-priori estimate

$$
\begin{aligned}
\|\vec{F}\|_{L^{p}\left(D^{2}\right)} & \leq C\|\vec{F}\|_{W^{1, q}} \leq C\left[\int_{D^{2}}|\nabla \vec{A}|^{q}|\nabla \vec{e}|^{q}+|\nabla \vec{B}|^{q}|\nabla \vec{e}|^{q}\right]^{\frac{1}{q}} \\
& \leq C\|\nabla \vec{A}\|_{p}\|\nabla \vec{e}\|_{2}+\|\nabla \vec{B}\|\|\nabla \vec{e}\|_{2} \\
& \leq C \varepsilon\left[\|\nabla \vec{A}\|_{p}+\|\nabla \vec{B}\|_{p}\right] .
\end{aligned}
$$

From this estimate, just as in the proof of Lemma A.2, we deduce the existence of $\vec{v}$ solving $\mathcal{L}_{\vec{n}} \vec{v}=\vec{g}$. Only now, $\vec{v}$ lies in $W_{0}^{1, p}$ for every $p>2$. Owing to the uniqueness result from Lemma A.2, it follows that every such $\vec{v}$ (one for each value of $p>2$ ) coincides with $\vec{\phi}$. Therefore, we find that $\vec{\phi} \in \bigcap_{p<+\infty} W_{0}^{1, p}\left(D^{2}\right)$.

Let now $x_{0}$ be a point in the interior of $D^{2}$. For any $\varepsilon>0$, we can find a radius $\rho>0$ such that

$$
\|\nabla \vec{n}\|_{L^{2,1}\left(B_{\rho}\left(x_{0}\right)\right)} \leq \varepsilon .
$$

Consider a smooth cut-off function $\chi$ equal to 1 on $B_{1 / 2}(0)$ and equal to 0 outside of $B_{1}(0)=D^{2}$. Let $\vec{w}$ be the function on the two-dimensional disk defined by:

$$
\vec{w}(x):=\vec{\phi}\left(\rho x+x_{0}\right) \chi(x) .
$$

Since $\vec{\phi} \in W^{1, p}\left(D^{2}\right)$ for every $p<+\infty$, in view of the computations (III.6)-(III.8), we infer the existence of $\vec{k} \in L^{2,1}\left(D^{2}\right)$ which satisfies:

$$
\begin{cases}\mathcal{L}_{\vec{n}} \vec{w}=\vec{k} & \text { in } D^{2} \\ \vec{w}=0 & \text { on } \partial D^{2} .\end{cases}
$$


We introduce now the Hodge decomposition of $\nabla \vec{w}-3 \pi_{\vec{n}}(\nabla \vec{w})=$ $\nabla \vec{C}+\nabla^{\perp} \vec{D}$ on $D^{2}$, with the boundary conditions $C=0$ on $\partial D^{2}$ and $\partial D / \partial v=0$ on $D^{2}$. From (A.64), it is not difficult to verify that

$$
\begin{cases}\Delta \vec{C}=\operatorname{div}\left(\vec{w} \wedge \nabla^{\perp} \vec{n}\right)+\vec{k} & \text { in } D^{2} \\ \vec{C}=0 & \text { on } \partial D^{2},\end{cases}
$$

and that

$$
\begin{cases}\Delta \vec{D}=3 \operatorname{div}\left(\pi_{\vec{n}}\left(\nabla^{\perp} \vec{w}\right)\right) & \text { in } D^{2} \\ \frac{\partial \vec{D}}{\partial \nu}=0 & \text { on } \partial D^{2} .\end{cases}
$$

Since the space of $L^{2}$ functions on $D^{2}$ with first derivatives in $L^{2,1}$ embeds in $L^{\infty}$, we derive the a-priori estimates

$$
\begin{aligned}
\|\nabla \vec{C}\|_{L^{\infty}\left(D^{2}\right)}+\left\|\nabla^{2} \vec{C}\right\|_{L^{2,1}\left(D^{2}\right)} & \leq C\|\Delta \vec{C}\|_{L^{2,1}\left(D^{2}\right)} \\
& \leq C\left[\|k\|_{L^{2,1}\left(D^{2}\right)}+\|\nabla \vec{n}\|_{L^{2,1}\left(D^{2}\right)}\|\nabla \vec{w}\|_{L^{\infty}}\right],
\end{aligned}
$$

and

$$
\begin{aligned}
\|\nabla \vec{D}\|_{L^{\infty}\left(D^{2}\right)}+\left\|\nabla^{2} \vec{D}\right\|_{L^{2,1}\left(D^{2}\right)} & \leq C\|\Delta \vec{D}\|_{L^{2,1}\left(D^{2}\right)} \\
& \leq C\|\nabla \vec{n}\|_{L^{2,1}\left(D^{2}\right)}\|\nabla \vec{w}\|_{L^{\infty}} .
\end{aligned}
$$

Note that we have used the fact that $\left|\operatorname{div}\left(\pi_{\vec{n}}(\nabla \vec{w})\right)\right| \leq C|\nabla \vec{n}||\nabla \vec{w}|$. Thus, choosing $\varepsilon$ small enough in (A.63), we can repeat the construction given in the proof of Lemma A.2 so as to infer the existence of a Lipschitz solution to (A.64) with second derivatives in $L^{2,1}$. The uniqueness result from Lemma A.1 ensures that this solution coincides with $\vec{w}$. Whence $\vec{\phi}\left(\rho x+x_{0}\right) \chi(x)$ is Lipschitz with second derivatives in $L^{2,1}$.

Taking into account the boundary condition $\vec{\phi}=0$, a similar argument can be developed for any point $x_{0}$ on the boundary of $D^{2}$. Doing so yields that $\vec{\phi}$ is Lipschitz with second derivatives in $L^{2,1}$, thereby completing the proof of Lemma A.7.

Lemma A.8 There exists $\varepsilon_{0}>0$ such that for every $0<\varepsilon<\varepsilon_{0}$ the following holds. Let $\vec{n}$ be a $W^{1,2}$ map from $D^{2}$ into the space of unit $(m-2)$ vectors in $\mathbb{R}^{m}$ such that

$$
\int_{D^{2}}|\nabla \vec{n}|^{2} d x \leq \varepsilon
$$

Assume further that $\nabla \vec{n}$ lies in the Lorentz space $L^{2,1}\left(D^{2}\right)$.

Let $\vec{v}$ be a function in $L^{2}\left(D^{2}\right)$ such that $\nabla \vec{v}$ is the sum of a compactly supported distribution in the open disk and of a function in $L^{2, \infty}\left(D^{2}\right)$ 
(in such a way that the trace of $\vec{v}$ on $\partial D^{2}$ is well defined). Suppose that $\vec{v}$ satisfies in the distributional sense the system

$$
\begin{cases}\Delta \vec{v}-3 \operatorname{div}\left(\pi_{\vec{n}}(\nabla \vec{v})\right)-\operatorname{div}\left(\vec{v} \wedge \nabla^{\perp} \vec{n}\right)=0 & \text { in } D^{2} \\ \vec{v}=0 & \text { on } \partial D^{2} .\end{cases}
$$

Then $\vec{v}$ vanishes identically in $D^{2}$.

Proof of Lemma A.8. We consider a smoothing $\vec{v}_{\delta}$ of $\vec{v}$ obtained through convolving with functions whose supports shrink approaching the boundary of $D^{2}$. As is easily seen, this ensures that $\nabla \vec{v}_{\delta} \in L^{2, \infty}\left(D^{2}\right)$, that $\vec{v}_{\delta}=\vec{v}$ in a neighborhood of $\partial D^{2}$, and that $\vec{v}_{\delta} \rightarrow \vec{v}$ in $L^{2}\left(D^{2}, \mathbb{R}^{m}\right)$. As in Sect. IV, let $\vec{\phi}_{i}$ be a sequence of normalized eigenvectors of $\mathscr{L}_{\vec{n}}$ in $W_{0}^{1,2}\left(D^{2}, \mathbb{R}^{m}\right)$, with corresponding eigenvalues $\lambda_{i}$ (these eigenvalues are nonzero, according to Lemma A.1). Recall that $\left\{\vec{\phi}_{i}\right\}$ forms an orthonormal Hilbert basis of $L^{2}\left(D^{2}, \mathbb{R}^{m}\right)$. By definition, we have

$$
\int_{D^{2}} \vec{v}_{\delta} \cdot \vec{\phi}_{i}=\lambda_{i}^{-1} \int_{D^{2}} \vec{v}_{\delta} \cdot \mathscr{L}_{\vec{n}} \vec{\phi}_{i}
$$

As explained in Lemma A.7, $\vec{\phi}_{i}$ is Lipschitz with $\nabla^{2} \vec{\phi}_{i} \in L^{2,1}$. Furthermore, $\nabla \vec{v}_{\delta} \in L^{2, \infty}$. And because both $\vec{\phi}_{i}$ and $\vec{v}_{\delta}$ vanish on $\partial D^{2}$, it follows that

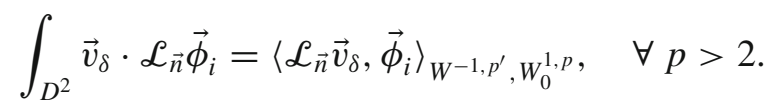

Note that $\Delta \vec{v}_{\delta}$ converges to $\Delta \vec{v}$ in $H^{-2}\left(D^{2}\right)$, the dual space of $W_{0}^{2,2}\left(D^{2}\right)$. In addition, $\operatorname{div}\left(\pi_{\vec{n}}\left(\nabla \vec{v}_{\delta}\right)\right)$ converges to $\operatorname{div}\left(\pi_{\vec{n}}(\nabla \vec{v})\right)$ in $W^{-1,1} \oplus H^{-2}\left(D^{2}\right)$, while $\operatorname{div}\left(\vec{v}_{\delta} \wedge \nabla^{\perp} \vec{n}\right)$ converges to $\operatorname{div}\left(\vec{v} \wedge \nabla^{\perp} \vec{n}\right)$ in $W^{-1,1}\left(D^{2}\right)$. Taking these facts into account, since $\vec{\phi}_{i}$ lies in $W^{1, \infty} \cap W^{2,2}\left(D^{2}\right)$, the duality appearing on the right-hand side of (A.72) becomes

$$
\int_{D^{2}} \vec{v} \cdot \mathcal{L}_{\vec{n}} \vec{\phi}_{i}=\left\langle\mathcal{L}_{\vec{n}}(\vec{v}), \vec{\phi}_{i}\right\rangle_{H^{-2} \oplus W^{-1,1}, W_{0}^{2,2} \cap W_{0}^{1, \infty},}
$$

as $\delta$ approaches zero.

Upon combining (A.70), (A.71), and (A.73), we deduce that $\int_{D^{2}} \vec{v} \cdot \vec{\phi}_{i}$ $=0$, for every $i \geq 1$. Whence, because $\left\{\vec{\phi}_{i}\right\}$ is a Hilbert basis for $L^{2}$, it follows that $\vec{v}$ vanishes identically in $L^{2}$. This concludes at once the proof of Lemma A.8.

With little effort, the proof of the previous lemma may be repeated mutatis mutandis so as to yield the following result.

Lemma A.9 There exists $\varepsilon_{0}>0$ such that for every $0<\varepsilon<\varepsilon_{0}$, a constant $C>0$ independent of $\varepsilon$ exists, with the following property. Let $\vec{n}$ 
be a Lipschitz map from $D^{2}$ into the space of unit $(m-2)$-vectors in $\mathbb{R}^{m}$ satisfying

$$
\int_{D^{2}}|\nabla \vec{n}|^{2} d x \leq \varepsilon .
$$

Suppose that $\vec{g}$ is a map in $L^{p}\left(D^{2}\right)$, for some $p>1$; and that $\vec{v}$ is an $L^{2}$ solution ${ }^{9}$ of

$$
\Delta \vec{v}-3 \operatorname{div}\left(\pi_{\vec{n}}(\nabla \vec{v})\right)-\operatorname{div}\left(\vec{v} \wedge \nabla^{\perp} \vec{n}\right)=0 \quad \text { in } D^{2} .
$$

Then there holds:

$$
\left\|\nabla^{2} \vec{v}\right\|_{L^{p}\left(D_{1 / 2}^{2}\right)} \leq C\left[\|\vec{g}\|_{L^{p}\left(D^{2}\right)}+\|\vec{v}\|_{L^{2}\left(D^{2}\right)}\right],
$$

where $D_{1 / 2}^{2}$ is the disk of radius $1 / 2$ centered on the origin.

\section{References}

[BR] Bernard, Y., Rivière, T.: Palais Smale sequences of the Willmore functional. In preparation (2008)

[Bla] Blaschke, W.: Vorlesungen über Differential Geometrie III. Springer, Berlin (1929)

[Bry] Bryant, R.L.: A duality theorem for Willmore surfaces. J. Differ. Geom. 20(1), 23-53 (1984)

[Che] Chen, B.-Y.: Some conformal invariants of submanifolds and their applications. Boll. Unione Mat. Ital. (4) 10, 380-385 (1974)

[CLMS] Coifman, R., Lions, P.-L., Meyer, Y., Semmes, S.: Compensated compactness and Hardy spaces. J. Math. Pures Appl., IX. Sér 72(3), 247-286 (1993)

[Fe] Federer, H.: Geometric Measure Theory. Springer, New York (1969)

[FJM] Friesecke, G., James, R.D., Müller, S.: A theorem on geometric rigidity and the derivation of nonlinear plate theory from three-dimensional elasticity. Commun. Pure Appl. Math. 55(11), 1461-1506 (2002)

[Haw] Hawking, S.W.: Gravitational radiation in an expanding universe. J. Math. Phys. 9, 598-604 (1968)

[Hel] Hélein, F.: Harmonic Maps, Conservation Laws, and Moving Frames. Camb. Tracts Math., vol. 150. Cambridge University Press, Cambridge (2002)

[Hef] Helfrich, W.: Elastic properties of lipid bilayers: theory and possible experiments. Z. Nat.forsch. A C28, 693-703 (1973)

[Hub] Huber, A.: On subharmonic functions and differential geometry in the large. Comment. Math. Helv. 32, 13-72 (1957)

[HI] Huisken, G., Ilmanen, T.: The Riemannian Penrose inequality. Int. Math. Res. Not. 1997(20), 1045-1058 (1997)

[KS1] Kuwert, E., Schätzle, R.: The Willmore flow with small initial energy. J. Differ. Geom. 57(3), 409-441 (2001)

[KS2] Kuwert, E., Schätzle, R.: Gradient flow for the Willmore functional. Commun. Anal. Geom. 10(2), 307-339 (2002)

[KS3] Kuwert, E., Schätzle, R.: Removability of point singularities of Willmore surfaces. Ann. Math. (2) 160(1), 315-357 (2004)

9 This $L^{2}$ condition is not optimal. It is however sufficient to our purpose. 
[LY] Li, P., Yau, S.T.: A new conformal invariant and its applications to the Willmore conjecture and the first eigenvalue of compact surfaces. Invent. Math. 69(2), 269-291 (1982)

[Mon] Montiel, S.: Willmore two-spheres in the four-sphere. Trans. Am. Math. Soc. 352(10), 4469-4486 (2000)

[MS] Müller, S., Sverák, V.: On surfaces of finite total curvature. J. Differ. Geom. 42(2), 229-258 (1995)

[PS] Pinkall, U., Sterling, I.: Willmore surfaces. Math. Intell. 9(2), 38-43 (1987)

[Ri1] Rivière, T.: Conservation laws for conformally invariant variational problems. Invent. Math. 168(1), 1-22 (2006)

[Ru] Rusu, R.: An algorithm for the elastic flow of surfaces. Interfaces Free Bound. 7, 229-239 (2005)

[Si1] Simon, L.: Lectures on Geometric Measure Theory. Proceedings of the Centre for Mathematical Analysis, Australian National University, vol. 3. Australian National University, Centre for Mathematical Analysis, Canberra (1983)

[Si2] Simon, L.: Existence of surfaces minimizing the Willmore functional. Commun. Anal. Geom. 1(2), 281-326 (1993)

[Sim] Simonett, G.: The Willmore flow near spheres. Differ. Integral Equ. 14(8), 10051014 (2001)

[Ta1] Tartar, L.: Remarks on oscillations and Stokes' equation. In: Macroscopic Modelling of Turbulent Flows (Nice, 1984). Lect. Notes Phys., vol. 230, pp. 24-31. Springer, Berlin (1985)

[Ta2] Tartar, L.: Imbedding theorems of Sobolev spaces into Lorentz spaces. Boll. Unione Mat. Ital., Sez. B, Artic. Ric. Mat. (8) 1(3), 479-500 (1998)

[Tho] Thomsen, G.: Über konforme Geometrie, I. Grundlagen der konformen Flächentheorie. Abh. Math. Semin. Univ. Hamburg, pp. 31-56 (1923)

[To1] Toro, T.: Surfaces with generalized second fundamental form in $L^{2}$ are Lipschitz manifolds. J. Differ. Geom. 39, 65-101 (1994)

[To2] Toro, T.: Geometric conditions and existence of bilipschitz parametrisations. Duke Math. J. 77(1), 193-227 (1995)

[Wei] Weiner, J.: On a problem of Chen, Willmore, et al. Indiana Univ. Math. J. 27(1), 19-35 (1978)

[Wen] Wente, H.C.: An existence theorem for surfaces of constant mean curvature. J. Math. Anal. Appl. 26, 318-344 (1969)

[Whi] White, J.H.: A global invariant of conformal mappings in space. Proc. Am. Math. Soc. 38, 162-164 (1973)

[Wil] Willmore, T.J.: Note on embedded surfaces. An. Ştiinţ. Univ. Al. I. Cuza Iaşi., Ser. Nouă, Mat. 11B, 493-496 (1965)

[Wil2] Willmore, T.J.: Riemannian Geometry. Oxford University Press, New York (1993) 